\title{
Packing cycles with modularity constraints
}

\author{
Paul Wollan *广 \\ Mathematisches Seminar der \\ Universität Hamburg \\ Bundesstr. 55 \\ 20146 Hamburg, Germany
}

\begin{abstract}
We prove that for all positive integers $k$, there exists an integer $N=N(k)$ such that the following holds. Let $G$ be a graph and let $\Gamma$ an abelian group with no element of order two. Let $\gamma: E(G) \rightarrow \Gamma$ be a function mapping elements of $\Gamma$ to the edges of $G$. A non-zero cycle is a cycle $C$ such that $\sum_{e \in E(C)} \gamma(e) \neq 0$ where 0 is the identity element of $\Gamma$. Then $G$ either contains $k$ vertex disjoint non-zero cycles or there exists a set $X \subseteq V(G)$ with $|X| \leq N(k)$ such that $G-X$ contains no non-zero cycle.

An immediate consequence is that for all positive odd integers $m$, a graph $G$ either contains $k$ vertex disjoint cycles of length not congruent to $0 \bmod m$, or there exists a set $X$ of vertices with $|X| \leq N(k)$ such that every cycle of $G-X$ has length congruent to 0 mod $m$. No such value $N(k)$ exists when $m$ is allowed to be even, as examples due to Reed and Thomassen show.
\end{abstract}

Key Words : Graph, Group labeling, Disjoint cycles, Erdős-Pósa Property

\section{Introduction}

Erdős and Pósa proved in [3] that there exists a function $f(k)$ such that for all positive integers $k$, either a graph contains $k$ vertex disjoint cycles or there exists a set $X$ of $f(k)$ vertices such that every cycle contains some vertex of $X$. We now say that a family of graphs $\mathcal{F}$ has the Erdös-Pósa property if there exists a function $f(k)$ such that every graph $G$ either has $k$ vertex disjoint subgraphs in $\mathcal{F}$ or there exists a set $X$ of at most $f(k)$ vertices such that every subgraph of $G$ isomorphic to a graph in $\mathcal{F}$ contains a vertex in $X$. Such a function $f$ will be referred to as an Erdös-Pósa function for the family $\mathcal{F}$, and such a set $X$ of vertices intersecting every subgraph in $\mathcal{F}$ is a hitting set.

An example of a family of graphs for which the Erdős-Pósa property does not hold is the class $\mathcal{C}_{\text {odd }}$, the set of cycles of odd length. This was first observed by Thomassen in [16]. Consider the graph constructed as follows. Start with the $k \times k$ grid for $k$ odd with the vertices labeled $v_{i, j}$ for $1 \leq i \leq k, 1 \leq j \leq k$, in the natural way. Add the edges $v_{1, i} v_{k, k-i+1}$ for $1 \leq i \leq k$ to form a projective planar graph $G_{k}$. The graph $G_{k}$ does not contain two disjoint odd cycles, but there do not exist $\lfloor k / 2\rfloor$ vertices intersecting every odd cycle. Reed [13] gave a related construction called Escher walls. Furthermore, Reed [13] gives a partial characterization of when a graph has neither many disjoint odd cycles nor a small hitting set for the set of odd cycles by showing that such graphs

*paul.wollan@gmail.com

${ }^{\dagger}$ This work partially supported by a fellowship from the Alexander von Humboldt Foundation 
must contain in a specific sense a large Escher wall. An immediate consequence of this result is that the Erdős-Pósa property holds for odd cycles in graphs embedded in any fixed orientable surface. Kawarabayashi and Nakamoto also independently showed that the Erdős-Pósa property holds for odd cycles in graphs embedded in any fixed orientable surface in [7]. In an alternate approach to the Erdős-Pósa property for odd cycles, Thomassen [17] showed that there exists a function $f(k)$ such that every $f(k)$-connected graph either contains $k$ disjoint odd cycles, or there exists a set $X$ of at most $2 k-2$ vertices hitting all odd cycles. Rautenbach and Reed proved that the function $f(k)$ can be chosen to be linear in $k$ in [12]. See [8] and [9] for further improvements on the connectivity bound.

In [2], Djeter and Neumann-lara give infinitely many pairs $l$ and $m$ such that the Erdös-Pósa property does not hold for the set of cycles of length $l$ mod $m$. They propose the question of classifying for which values $l$ and $m$ does the family of cycles of length $l \bmod m$ have the Erdös-Pósa property. The projective planar graph $G_{k}$ defined in the previous paragraph can be modified to show that the Erdös-Pósa does not hold for the class of cycles of length $l \bmod m$ for all fixed even $m$ and $0<l<m$ such that $l$ is odd. To see this, fix such $m$ and $l$ and subdivide every edge of the grid to form a path of length $m$ and subdivide every edge of the form $v_{1, i} v_{k, k-i+1}$ to form a path of length $l$. In the subdivided graph, every cycle of length $l \bmod m$ must use an odd number of the subpaths of length $l$. It follows that there do not exist even two disjoint cycles of length $l \bmod m$, and yet, there do not exist $\lfloor k / 2\rfloor$ vertices intersecting every cycle of length $l \bmod m$. Thomassen showed that for all positive integers $m$, the class of cycles of length $0(\bmod m)$ does have the Erdős-Pósa property in $[16]$. We give the following theorem.

Theorem 1.1 There exists a function $f(k)$ such that the following holds. For all positive integers $k$ and all positive odd integers $m$, a graph $G$ either has $k$ disjoint cycles of non-zero length mod $m$, or there exists a set $X \subseteq V(G)$ of size at most $f(k)$ hitting all such cycles.

As above, the example $G_{k}$ can be modified to show that the family of cycles of non-zero length mod $m$ for a fixed even integer $m$ does not have the Erdös-Pósa property. To see this, we subdivide each edge of the grid to be a path of length $m$ and we subdivide the edges of the form $v_{1, i} v_{k, k-i+1}$ to form a path of length $m / 2$. Such a graph does not contain two disjoint cycles of non-zero length mod $m$, nor does it contain a small hitting set for all such cycles.

One might object that the construction is only 2-connected and has many vertices of degree two. However, adding a single vertex $v$ adjacent to every other vertex of the subdivided $G_{k}$ yields a 3-connected graph that again serves as a counter-example to the Erdős-Pósa property holding for both the family of non-zero cycles $\bmod m$ when $m$ is even and the family of cycles of length $l$ mod $m$ for $m$ even and $0<l<m, l$ odd.

To prove Theorem 1.1, we will actually prove a more general statement concerning group labeled graphs. Let $\Gamma$ be an abelian group. As we will be considering abelian groups in this article, we will use the addition notation for groups with 0 indicating the identity element of a group. A $\Gamma$-labeling of a graph $G$ is a function $\gamma: E(G) \rightarrow \Gamma$. For any subgraph $H$ of $G$, we let $\gamma(H)=\sum_{e \in E(H)} \gamma(e)$. A non-zero cycle in a $\Gamma$-labeled graph is a cycle $C$ such that $\gamma(C) \neq 0$. Recall that the order of any element $\alpha \neq 0$ of a group $\Gamma$ is the minimum $n$ such that $n \alpha=0$. We prove the following.

Theorem 1.2 There exist constants $c$ and $c^{\prime}$ such that the following holds. Let $G$ be a $\Gamma$-labeled graph where $\Gamma$ does not have any elements of order two. Then for all positive integers $k$, either $G$ 
contains $k$ disjoint non-zero cycles, or there exists a set $X \subseteq V(G)$ with $|X| \leq c^{k^{c^{\prime}}}$ such that $G-X$ does not contain any non-zero cycles.

Theorem 1.1 is an immediate consequence of Theorem 1.2 as we can consider cycles of non-zero length mod $m$ to be non-zero cycles in the graph labeled by the group $\mathbb{Z}_{m}$ with every edge having weight one.

We observe that the condition that $\Gamma$ be an abelian group is necessary; otherwise the idea of non-zero cycles is not well defined. Consider a graph labeled with elements from a non-abelian group $\Gamma$. Let $C$ be a 3 -cycle with edges $e_{1}, e_{2}$, and $e_{3}$ labeled $\alpha, \beta$, and $\alpha^{-1} \beta^{-1}$, respectively. If we calculate the weight of the subgraph traversing the edges $e_{1}, e_{3}, e_{2}$ in that order, then the weight of the cycle would be equal to the identity. However, if we calculate the weight of the cycle traversing the edges $e_{1}, e_{2}, e_{3}$, the weight would be $\alpha \beta \alpha^{-1} \beta^{-1}$ which need not be equal to the identity.

We will need the following basic definitions. A linkage is a graph where every component is a path, and we say that a path links its endpoints. If $A$ is a subset of the vertices of a graph $G$, then a non-trivial path intersecting $A$ in exactly its endpoints is an $A$-path. If $P$ is a path with endpoints $x$ and $y$, the interior of the path, denoted $\operatorname{int}(P)$, is the vertex set $V(P)-\{x, y\}$. Given two vertices $a$ and $b$ in a path $P$, the subpath of $P$ linking $a$ and $b$ is denoted $a P b$. If $G$ and $H$ are two graphs, we denote the graph with vertex set $V(G) \cup V(H)$ and edge set $E(G) \cup E(H)$ by $G \cup H$. Similarly, we denote by $G \cap H$ the graph with vertex set $V(G) \cap V(H)$ and edge set $E(G) \cap E(H)$. For any graph $G$ of minimum degree two, the branch vertices of $G$ is the set of vertices of degree at least three. A segment of $G$ is a non-trivial path $P$ in $G$ such that $P$ intersects the branch vertices exactly in its endpoints.

As a technicality, we extend the definition of the Erdős-Pósa property to group labeled graphs in the natural way. If $\mathcal{F}$ is a family of $\Gamma$-labeled graphs, then $\mathcal{F}$ has the Erdös-Pósa property with Erdős-Pósa function $f$ if for all $k \geq 1$, every $\Gamma$-labeled graph $G$ either has $k$ disjoint subgraphs isomorphic to a graph in $\mathcal{F}$, or there exists a set $X \subseteq V(G)$ such that $G-X$ contains no subgraph isomorphic to a graph in $\mathcal{F}$. Here the isomorphism must respect the group values assigned to the edges.

The proof of Theorem 1.2 proceeds as follows. By choosing a huge function as a potential Erdős-Pósa function, we can ensure that a minimal group labeled counterexample $G$ must contain a subgraph $H$ isomorphic to a subdivision of a large grid-like graph called a wall. Moreover, the subgraph $H$ is highly linked to every non-zero cycle of $G$. That is, if we let $X$ be the vertices of $H$ of $\operatorname{deg}_{H}$ at least three, there does not exist a small cut separating $X$ from any non-zero cycle $C$. This argument is laid out in Section 2. A result of [18] implies that there exists a collection of disjoint paths $P_{1}, \ldots, P_{m}$ for some large integer $m$, such that each $P_{i}$ intersects $X$ exactly in its endpoints, and, moreover, every path $P_{i}$ has non-zero weight. The remainder of the argument consists in proving the claim, stated as Theorem 3.1, that if $m$ is sufficiently large with respect to $k$, then the wall along with the non-zero paths $P_{1}, \ldots, P_{m}$ must contain $k$ disjoint non-zero cycles. The proof of Theorem 1.2 (assuming Theorem 3.1) is given in Section 3. The proof of Theorem 3.1 comprises the main difficulties of the argument. After some preliminary lemmas presented in Section 4, the proof of Theorem 3.1 is given in Section 5 .

\section{Tangles and the Erdős-Pósa property}

The proof of Theorem 1.2 relies heavily on the idea of a tangle, introduced in [14]. A separation of a graph $G$ is a pair of subgraphs $(A, B)$ such that $A \cup B=G$. The $\operatorname{order}$ of a separation $(A, B)$ is 
$|A \cap B|$. If $t \geq 1$ is a positive integer, a tangle of order $t$ in a graph $G$ is a set $\mathcal{T}$ of ordered pairs such that

1. if $(A, B)$ is a separation of $G$ of order $<t$, then $\mathcal{T}$ contains one of $(A, B),(B, A)$,

2. if $\left(A_{1}, B_{1}\right),\left(A_{2}, B_{2}\right)$, and $\left(A_{3}, B_{3}\right) \in \mathcal{T}$, then $A_{1} \cup A_{2} \cup A_{3} \neq G$,

3. and if $(A, B) \in \mathcal{T}$ then $V(A) \neq V(G)$.

Tangles arise in a natural way when considering the Erdős-Pósa property for a given family of connected graphs.

Let $\mathcal{F}$ be a family of graphs, possibly group labeled, and let $f$ be a function. The pair $(G, k)$ forms a counterexample to $f$ being an Erdős-Pósa function for $\mathcal{F}$ if the graph $G$ neither contains $k$ disjoint subgraphs each isomorphic to some graph in $\mathcal{F}$ nor contains a hitting set $X \subseteq V(G)$ for the family $\mathcal{F}$ with $|X| \leq f(k)$. If $\mathcal{F}$ is a family of $\Gamma$-labeled graphs for some group $\Gamma$, then $G$ must also be a $\Gamma$-labeled graph.

Lemma 2.1 Let $\mathcal{F}$ be a family of connected graphs, possibly group labeled. Let $f$ be a positive function that is not an Erdös-Pósa function for $\mathcal{F}$, and let $(G, k)$ be a counter-example to $f$ being an Erdös-Pósa function for $\mathcal{F}$ with $k$ chosen minimal over all such counter-examples. Let $t$ be a positive integer such that $t \leq f(k)-2 f(k-1)$ and $t \leq f(k) / 3$, and let $\mathcal{T}$ be the set of all ordered pairs $(A, B)$ where $(A, B)$ is a separation of order at most $t$ such that $B$ contains a subgraph in $\mathcal{F}$. Then $\mathcal{T}$ forms a tangle in $G$ of order $t$.

Proof. We first claim that for any separation $(A, B)$ of order less than $t$ in $G$, at most one of $A$ and $B$ can contain a subgraph contained in $\mathcal{F}$. Assume, to reach a contradiction, that both $A$ and $B$ contain a subgraph in $\mathcal{F}$. Lest $G$ contain $k$ disjoint subgraphs in $\mathcal{F}$, there do not exist $k-1$ disjoint subgraphs in $\mathcal{F}$ in either the graph $A-V(B)$ or the graph $B-V(A)$. It follows by our choice of $(G, k)$ to minimize $k$ that there exists a set $X_{A} \subseteq V(A)$ with $\left|X_{A}\right| \leq f(k-1)$ and a set $X_{B} \subseteq V(B)$ with $\left|X_{B}\right| \leq f(k-1)$ such that every subgraph of $\mathcal{F}$ contained in $G$ intersects the set $X:=X_{A} \cup X_{B} \cup(V(A) \cap V(B))$. Moreover, $|X| \leq f(k)$, a contradiction to our choice of $k$ and $G$. We conclude that if $(A, B) \in \mathcal{T}$, then $(B, A) \notin \mathcal{T}$. Note that this argument relies on our choice of $\mathcal{F}$ to only contain connected graphs. Also, if $(A, B)$ is a separation of order at most $t$, then at least one of $A$ and $B$ must contain a subgraph of $\mathcal{F}$ by our choice of $(G, k)$ to form a counter-example. Thus 1 . is satisfied in the definition of tangle.

Property 2. and 3. in the definition of tangle are satisfied by our assumption that $t \leq f(k) / 3$ and the fact that $A$ cannot contain any subgraph of $\mathcal{F}$ for any separation $(A, B)$ in $\mathcal{T}$.

We say that $G$ contains a $g \times g$ grid minor if there exist pairwise disjoint sets $\left\{X_{i, j}: 1 \leq i \leq\right.$ $g, 1 \leq j \leq g\}$ and edges $\left\{e_{i, j}^{\text {right }} \in E(G): 1 \leq i \leq g, 1 \leq j \leq g-1\right\} \cup\left\{e_{i, j}^{\text {down }}: 1 \leq i \leq g-1,1 \leq j \leq g\right\}$ such that $G\left[X_{i, j}\right]$ is connected, the edge $e_{i, j}^{\text {right }}$ has one end in $X_{i, j}$ and one end in $X_{i, j+1}$, and the edge $e_{i, j}^{\text {down }}$ has one end in $X_{i, j}$ and one end in $X_{i+1, j}$. Let $H$ be the subgraph of $G$ comprising a $g \times g$ grid minor. The $i^{t h}$ row of $H$ is the set of edges $\left\{e_{i, j}^{\text {right }}: 1 \leq j \leq g-1\right\}$ for some fixed $i$. Let $\mathcal{T}_{H}$ be the set of all separations $(A, B)$ of order $<g$ such that $E(B)$ includes a row of $H$. It follows from a result of [14] (Theorem 7.3) that $\mathcal{T}_{H}$ is a tangle in $G$ of order $g$. A tangle $\mathcal{T}$ dominates $H$ if $\mathcal{T}_{H} \subseteq \mathcal{T}$

The following theorem of Robertson, Seymour, and Thomas [15] gives an improved explicit bound to a similar qualitative result appearing in [14]. 
Theorem 2.2 Let $g \geq 2$ and let $\mathcal{T}$ be a tangle in $G$ of order $\geq 20^{g^{4}(2 g-1)}$. Then $\mathcal{T}$ dominates a $g \times g$ grid minor.

Combining Theorem 2.2 with Lemma 2.1, we will see that if we are looking for an Erdös-Pósa function for a particular family $\mathcal{F}$ of graphs, then an appropriately minimal counter-example $G$ will not only have a large grid minor, but that grid minor will be well connected to every subgraph of $\mathcal{F}$ contained in $G$. We make this idea explicit below as Theorem 2.3.

For the proofs to come, we will work with a variation of a grid minor that will allow us to consider a subdivision instead of a minor. We define the graph $W_{l, m}$ the $l \times m$ wall. Let $V\left(W_{l, m}\right)=\left\{v_{i, j}\right.$ : $1 \leq i \leq l, 1 \leq j \leq m\}$ and

$$
\begin{aligned}
E\left(W_{l, m}\right)= & \left\{v_{i, j} v_{i, j+1}: 1 \leq i \leq l, 1 \leq j \leq m-1\right\} \\
& \cup\left\{v_{2 i-1,2 j-1} v_{2 i, 2 j-1}: 1 \leq i \leq l / 2,1 \leq j \leq\lceil m / 2\rceil\right\} \\
& \cup\left\{v_{2 i, 2 j} v_{2 i+1,2 j}: 1 \leq i \leq l-1 / 2,1 \leq j \leq m / 2\right\}
\end{aligned}
$$

Equivalently, $W_{l, m}$ consists of $l$ disjoint paths $P_{1}, \ldots, P_{l}$ each containing $m$ vertices such that for odd $i$, the odd vertices of $P_{i}$ and $P_{i+1}$ are adjacent, and for even $i$, the even vertices of $P_{i}$ and $P_{i+1}$ are adjacent. It is easy to see that if a graph $G$ contains a $g \times g$ grid minor, then $G$ contains a subgraph isomorphic to a subdivision of the $g \times g$ wall. Moreover, the subdivision contains every row of the grid minor.

Theorem 2.3 Let $\mathcal{F}$ be a family of connected, possibly group labeled, graphs. For all positive integers $k$, let $l=l(k), m=m(k)$, and $t=t(k)$ be positive integers satisfying $l(k) \geq t(k)$ and $m(k) \geq t(k)$. For all $k$, let $\alpha(k)=\max \{m(k), t(k)+1, l(k)\}$. Define the function

$$
f(k)=4^{k} 20^{\alpha(k)^{4}(2 \alpha(k)-1)} .
$$

Assume $f$ is not an Erdös-Pósa function for $\mathcal{F}$, and let $(G, k)$ be a counter-example with $k$ chosen minimal among all such counter-examples. Then there exists a subgraph $H$ of $G$ isomorphic to a subdivision of the $l \times m$ wall such that for every graph $F$ of $G$ isomorphic to a graph of $\mathcal{F}$, there exist $t$ vertex disjoint paths linking the branch vertices of $H$ and $V(F)$ in $G$.

Proof. Let $\mathcal{F}, l, m, t, \alpha$, and $f$ be given. Let $(G, k)$ be a counter-example to $f$ being an Erdős-Pósa function for the family $\mathcal{F}$, and assume it is chosen from all such counter-examples to minimize $k$. Let $\mathcal{T}$ be all the separations $(A, B)$ of order at most $20^{\alpha(k)^{4}(2 \alpha(k)-1)}$ such that $B$ contains a subgraph in $\mathcal{F}$. Lemma 2.1 implies that $\mathcal{T}$ is a tangle of order $20^{\alpha(k)^{4}(2 \alpha(k)-1)}$. Theorem 2.2 implies that there exists an $\alpha \times \alpha$ grid minor $H$ such that $\mathcal{T}$ dominates $H$. As we observed above, $H$ contains a subgraph $H^{\prime}$ isomorphic to a subdivision of the $l \times m$ wall. Let the branch vertices of $H^{\prime}$ be labeled $v_{i, j}$ in the natural way. We may assume that the path in $H^{\prime}$ linking $v_{i, j}$ and $v_{i, j+1}$ that corresponds to the edge $v_{i, j} v_{i, j+1}$ in the $l \times m$ wall contains exactly one edge of the $i^{t h}$ row of the $\alpha \times \alpha$ grid minor.

Let $X$ be the vertices of $H^{\prime}$ of $\operatorname{deg}_{H^{\prime}}$ at least three. Observe that if we let $X_{i, j}$ be as in the definition of the $\alpha \times \alpha$ grid minor, then every vertex $v_{i, j}$ of $X$ is contained in $X_{i, j}$. It follows that for any row of $H$, if we let $Y$ be the endpoints of the edges comprising the row, then there exist at least $t+1$ disjoint paths linking $Y$ to $X$. Assume the theorem is false and that there exists a subgraph $D$ of $G$ contained in $\mathcal{F}$, but that there do not exist $t$ disjoint paths linking $D$ and $X$. Then there exists a separation $(A, B)$ of order at most $t-1$ with $X \subseteq V(A)$ and $D \subseteq V(B)$. The separation $(A, B)$ is 
contained in $\mathcal{T}$, and consequently, in $\mathcal{T}_{H}$. It follows that there exists an index $i$ such that the $i^{\text {th }}$ a row of the $\alpha \times \alpha$ grid minor contained in $B$. But this is a contradiction, as we saw above, since there exist $t+1$ disjoint paths linking the row and the set $X$. This completes the proof of the theorem.

\section{Proof of Theorem 1.2}

In the proof of Theorem 1.2, we utilize Theorem 2.3 to ensure that a potential counter-example to Theorem 1.2 has a subdivided wall which we can make as large as we need by choosing a sufficiently large Erdős-Pósa function. The next theorem allows us to find many non-zero cycles if we can find many non-zero paths attaching to the branch vertices of the wall.

Theorem 3.1 There exists a constant $c$ such that for all integers $k \geq 1$ the following is true. Let $n_{k}=c k^{87}$ and let $l \geq n_{k}, m \geq n_{k}$ be positive integers such that $l$ is congruent to 0 mod 4 and $m$ is congruent to $7 \bmod 8$. Let $\Gamma$ be an abelian group not containing any elements of order two, and let $G$ be a $\Gamma$-labeled graph with weight function $\gamma$. Let $H$ be a subgraph of $G$ isomorphic to a subdivision of $W_{l, m}$ and let $X$ be the vertices of $H$ of degree at least three. If $G$ contains $n_{k}$ disjoint $X$-paths $P_{1}, \ldots, P_{n_{k}}$ such that $\gamma\left(P_{i}\right) \neq 0$ for $1 \leq i \leq n_{k}$, then $G$ contains $k$ vertex disjoint cycles of non-zero weight.

The proof of Theorem 3.1 is somewhat technical, and we leave the proof for Section 5 . Note that if $l$ is even and $m$ is odd, the $l \times m$ wall has minimum degree two. Throughout the proofs, assuming that a given wall has minimum degree two will simplify the notation and avoid the technicality of repeatedly deleting vertices of degree one. Thus, our choice of $l$ and $m$ to satisfy the given modularity constraints is merely a convenience, and not of fundamental importance to the results.

In order to find many such non-zero paths attaching to the branch vertices, we need the following result from [18].

Theorem 3.2 There exists a constant $c$ such that the following holds. Let $\Gamma$ be an abelian group and let $\gamma$ be a $\Gamma$-labeling of a graph $G$. Let $A \subseteq V(G)$ be a fixed set of vertices of $G$. Then either there exist disjoint $A$-paths $P_{1}, P_{2}, \ldots, P_{k}$ with $\gamma\left(P_{i}\right) \neq 0$, or there exists a set $X \subseteq V(G)$ with $|X| \leq c k^{4}$ such that $G-X$ has no non-zero $A$ path.

We are now reach to give the proof of Theorem 1.2.

Proof. (Theorem 1.2, assuming Theorem 3.1) Let $c_{1}$ be the constant in the statement of Theorem 3.2. Let $c_{2}$ be the constant in Theorem 3.1. We set $t(k):=c_{1}\left(c_{2} k^{87}\right)^{4}+3, l(k)=4\lceil t(k) / 4\rceil$ and $m(k)=8\lceil t(k) / 8\rceil+7$. We set $\alpha(k)=\max \{l(k), m(k), t(k)+1\}$. Fix $c$ and $c^{\prime}$ such that

$$
c^{k^{c^{\prime}}} \geq 4^{k} 20^{\alpha(k)^{4}(2 \alpha(k)-1)} \text {. }
$$

We claim $c$ and $c^{\prime}$ satisfy the statement of Theorem 1.2. Assume not. Then there exists a group $\Gamma$ with no element of order two, a graph $G$, a positive integer $k$, and a $\Gamma$-labeling $\gamma$ of $G$ such that $G$ neither has $k$ disjoint non-zero cycles, nor does there exist a covering set $X$ of size at most $c^{k^{c^{\prime}}}$. Assume that we chose $G, \Gamma$, and $k$ to minimize the value of $k$. By Theorem 2.3, there exists a subgraph $H$ of $G$ isomorphic to a subdivision of the $l \times m$ wall such that there exist $t(k)$ disjoint paths linking the branch vertices of $H$ to any non-zero cycle in $G$. Let $X$ be the branch vertices of $H$. By Theorem 3.1, there do not exist $c_{2} k^{87}$ disjoint non-zero $X$-paths. Consequently, by Theorem 
3.2, there exists a set $Z$ of vertices with $|Z| \leq c_{1}\left(c_{2} k^{87}\right)^{4}$ such that $G-Z$ does not contain any non-zero $X$-path.

We claim the set $Z$ intersects every non-zero cycle in $G$. This will contradict our choice of $G$ to be a counter-example, as $|Z| \leq c^{k^{c^{\prime}}}$. If $Z$ is not a hitting set, then there exists a non-zero cycle $C$ disjoint from $Z$. There exist $t(k)$ disjoint paths linking $X$ to $C$. It follows that there exist three disjoint paths from $C$ to $X$ avoiding $Z$, call them $P_{1}, P_{2}$, and $P_{3}$. We claim that $P_{1} \cup P_{2} \cup P_{3} \cup C$ contains a non-zero $X$-path, contrary to the fact that $Z$ intersects every such non-zero $X$-path. To see this, for $i=1,2,3$, let $Q_{i}$ be the subpath in $C$ linking the endpoints of $P_{i}$ and $P_{i+1}$ avoiding $P_{i+2}$ where all the index notation is taken $\bmod 3$. Let $a_{i}=\gamma\left(P_{i}\right)$ for $i=1,2,3$. Lest $P_{i} \cup Q_{i} \cup P_{i+1}$ contain a non-zero $X$-path, we see $\gamma\left(Q_{i}\right)=-a_{i}-a_{i+1}$ for $i=1,2,3$. Adding the terms together, we get that $\gamma(C)=-2 a_{1}-2 a_{2}-2 a_{3}$. Similarly, lest $P_{i} \cup Q_{i-1} \cup Q_{i+1} \cup P_{i+1}$ contain a non-zero $X$-path, we see that $\gamma\left(Q_{i-1}\right)+\gamma\left(Q_{i+1}\right)=\gamma\left(Q_{i}\right)=-a_{i}-a_{i+1}$ for $i=1,2,3$. This implies that $\gamma(C)=-2 a_{i}-2 a_{i+1}$. Merging the equations, we see that $-2 a_{i}-2 a_{i+1}=-2 a_{1}-2 a_{2}-2 a_{3}$ for $i=1,2,3$. It follows that $2 a_{i}=0$ for $i=1,2,3$. Consequently, $\gamma(C)=-2 a_{1}-2 a_{2}-2 a_{3}=0$, a contradiction. We conclude that $P_{1} \cup P_{2} \cup P_{3} \cup C$ in fact does contain a non-zero $X$-path. This final contradiction to our choice of $Z$ completes the proof of the theorem.

\section{Non-zero paths attaching to ladders and grids}

We begin with several preliminary results before proceeding with the proof of Theorem 3.1 in the next section. Let $G$ be a $\Gamma$-labeled graph with weight function $\gamma$ for some group $\Gamma$. A non-zero theta is a subgraph of $G$ consisting of three internally disjoint paths $P_{1}, P_{2}, P_{3}$ each with endpoints equal to $x$ and $y$ for some pair of vertices $x, y$ and furthermore, such that there exists an index $i \in\{1,2,3\}$ such that $\gamma\left(P_{i}\right) \neq 0$.

Observation 1 Let $G$ be a $\Gamma$-labeled graph with weight function $\gamma$. If $\Gamma$ does not contain an element of order two, then every non-zero theta contains a non-zero cycle.

Proof. Let $P_{1}, P_{2}, P_{3}, G$ and $\Gamma$ be as in the statement. Without loss of generality, assume $\gamma\left(P_{1}\right)=\alpha$, an element of $\Gamma$ which has order not equal to two. Lest $P_{1} \cup P_{2}\left(P_{1} \cup P_{3}\right)$ form a non-zero cycle, we see that $\gamma\left(P_{2}\right)=\gamma\left(P_{3}\right)=-\alpha$. By our choice of $\alpha$, the graph $P_{2} \cup P_{3}$ is a non-zero cycle, proving the observation.

We will need the following classic result of Erdős and Szekeres.

Theorem 4.1 [3] Let $a=\left(a_{1}, a_{2}, \ldots, a_{n}\right)$ be a sequence of positive, distinct integers. If $n \geq k^{2}$, then a either contains a strictly increasing subsequence of length $k$ or a strictly decreasing subsequence of length $k$.

From Theorem 4.1, we conclude the following lemma.

Lemma 4.2 Let $P_{1}$ and $P_{2}$ be two vertex disjoint paths in a $\Gamma$-labeled graph $G$ with weight function $\gamma$. Assume that $\Gamma$ does not contain any elements of order two. Let $Q_{1}, \ldots, Q_{l}$ be vertex disjoint paths in $G$ such that $\gamma\left(Q_{i}\right)$ is non-zero and each $Q_{i}$ has one endpoint in $P_{1}$ and one endpoint in $P_{2}$ for $i=1, \ldots, l$. If

$$
l \geq 9 k^{2}
$$


then $G$ contains $k$ vertex disjoint non-zero cycles.

Proof. Let $G, P_{1}, P_{2}, \Gamma$, and $Q_{1}, \ldots, Q_{l}$ be as in the statement. We will prove that $G$ contains $k$ disjoint non-zero cycles by proving that $G$ in fact contains $k$ disjoint non-zero thetas. Label the vertices of $P_{1} \cap\left(\bigcup_{i} V\left(Q_{i}\right)\right)$ by $1,2, \ldots, n$ so that they occur on the path $P_{1}$ in that order, and similarly, let the vertices of $P_{2} \cap\left(\bigcup_{i} V\left(Q_{i}\right)\right)$ be labeled $1^{\prime}, 2^{\prime}, \ldots, n^{\prime}$ so that they occur in that order on the path $P_{2}$. We define a sequence $a(1), a(2), \ldots, a(n)$ such that for all $i, 1 \leq i \leq n$, there exists an index $j$ such that $Q_{j}$ has endpoints $i$ in $P_{1}$ and $a(i)^{\prime}$ in $P_{2}$. By Theorem 4.1, there exists values $1 \leq \pi_{1}<$ $\pi_{2}<\cdots<\pi_{3 k} \leq n$ such that the subsequence $a\left(\pi_{1}\right), a\left(\pi_{2}\right), \ldots, a\left(\pi_{3 k}\right)$ is either monotone increasing or decreasing. Let $\sigma_{1}, \sigma_{2}, \ldots, \sigma_{3 k}$ be such that $Q_{\sigma_{i}}$ has endpoints $\pi_{i}$ and $a\left(\pi_{i}\right)^{\prime}$ for $1 \leq i \leq 3 k$. Then for all $t, 2 \leq t \leq 3 k-1$, the subgraph $\pi_{t-1} P_{1} \pi_{t+1} \cup a\left(\pi_{t-1}\right)^{\prime} P_{2} a\left(\pi_{t+1}\right)^{\prime} \cup Q_{\sigma_{t-1}} \cup Q_{\sigma_{t}} \cup Q_{\sigma_{t+1}}$ forms a non-zero theta. Thus we have constructed $k$ distinct non-zero thetas and they will be disjoint by the fact that the subsequence $a\left(\pi_{1}\right), a\left(\pi_{2}\right), \ldots, a\left(\pi_{3 k}\right)$ is monotone.

The following related lemma is an immediate consequence of a theorem of Rado [11] concerning sets of intervals.

Lemma 4.3 [11] Let $I_{1}, I_{2}, \ldots, I_{k^{2}}$ be subintervals of $[0,1]$. Then either there exists a subcollection of $k$ pairwise disjoint intervals or $k$ pairwise intersecting intervals.

Lemma 4.4 follows easily from Lemma 4.3. We omit the proof here.

Lemma 4.4 Let $P$ be a path and let $Q_{1}, \ldots, Q_{n}$ be disjoint $V(P)$-paths. If $n \geq k^{2}$, then one of the following outcomes holds.

1. There exists a subset $I \subseteq\{1,2, \ldots, n\}$ with $|I| \geq k$ and an edge $e \in V(P)$ such that for every $i \in I$, the path $Q_{i}$ has one endpoint in each of the two components of $P-e$, or

2. there exists a subset $I \subseteq\{1,2, \ldots, n\}$ with $|I| \geq k$ such that for every distinct $i, j \in I$, the subpath of $P$ linking the ends of $Q_{i}$ is disjoint from the subpath of $P$ linking the ends of $Q_{j}$.

Before proceeding to analyze the situation when we have many non-zero paths attaching to a subdivision of a grid, we first consider a simpler graph. The $k$ ladder, denoted $L_{k}$ is the graph with vertex set $\left\{v_{1}, v_{2}, \ldots, v_{k}, u_{1}, u_{2}, \ldots, u_{k}\right\}$ and edges $\left\{v_{i} v_{i+1}: 1 \leq i \leq k-1\right\} \cup\left\{u_{i} u_{i+1}: 1 \leq i \leq\right.$ $k-1\} \cup\left\{u_{i} v_{i}: 1 \leq i \leq k\right\}$. In other words, the $k$ ladder is formed by taking two paths of length $k-1$ and joining the corresponding vertices on the paths with an edge.

Lemma 4.5 Let $G$ be a $\Gamma$-labeled graph with weight function $\gamma$. Assume $\Gamma$ does not contain any elements of order two. Let $H$ be a subgraph of $G$ isomorphic to a subdivision of an l-ladder for $l \geq 3$. Let $X$ be the branch vertices of $H$. Let $P_{1}, \ldots, P_{n}$ be non-zero $V(H)$-paths such that no $P_{i}$ has both endpoints contained in a segment of $H$. For all positive integers $k$, if

$$
n \geq 27816 k^{6} \geq\left[3\left(9 k^{2}\right)^{2}+(3 k+2)\right]\left(6\left(18 k^{2}+2\right)\right),
$$

then $G$ contains $k$ vertex disjoint non-zero cycles.

Proof. Let $G, H, \Gamma, \gamma$, and $P_{1}, \ldots, P_{n}$ be as in the statement. Let $X$ be the branch vertices of $H$. Assume the lemma is false, and let $G$ be a counter-example. 
First, assume there exists a segment $P$ of $H$ such that $P$ contains the endpoints of $18 k^{2}+2$ distinct paths $P_{i}$. Let $Z$ be the internal vertices of $P$. There are then $18 k^{2}$ distinct paths $P_{i}$ with one endpoint in the set $Z$. Observe that there exist two (not necessarily disjoint) paths in $H-Z$ covering the vertices of $H-Z$. It follows from Lemma 4.2 that there exists a path $P^{\prime}$ in $H-Z$ such for at least $9 k^{2}$ distinct indices $i$, the path $P_{i}$ has one endpoint in $P$ and one endpoint in $P^{\prime}$. Lemma 4.2 implies that $G$ contains $k$ vertex disjoint cycles, a contradiction to our choice of $G$.

We conclude that there exists a subset $I \subseteq\{1,2, \ldots, n\}$ such that for any $i, j \in I$, there does not exist a segment of $H$ containing endpoints of both $P_{i}$ and $P_{j}$. Since for every path $P_{i}$ there are at most six distinct segments of $H$ containing an endpoint of $P_{i}$, and combined with the arguments of the previous paragraph, we see that $|I| \geq n /\left(6\left(18 k^{2}+2\right)\right) \geq\left[3\left(9 k^{2}\right)^{2}+(3 k+2)\right]$.

For the remainder of the proof, we will need to refer more specifically to the segments and branch vertices of $X$. The vertices of the $l$-ladder are labeled $\left\{u_{1}, u_{2}, \ldots, u_{l}, v_{1}, v_{2}, \ldots, v_{l}\right\}$. We naturally label the branch vertices $X$ of $H$ with $\left\{u_{2}, u_{3}, \ldots, u_{l-1}, v_{2}, \ldots, v_{l-1}\right\}$. We label the segments of $H$ as follows. Let the segments $Q_{2}, Q_{3}, \ldots, Q_{l-2}$ be defined where $Q_{i}$ has endpoints $u_{i}$ and $u_{i+1}$ for all $2 \leq i \leq l-2$. Let the segments $R_{2}, R_{3}, \ldots, R_{l-2}$ be defined where $R_{i}$ has endpoints $v_{i}$ and $v_{i+1}$ for $2 \leq i \leq l-2$. Let $S_{1}, S_{2}, \ldots, S_{l}$ be defined where $S_{i}$ has endpoints $u_{i}$ and $v_{i}$ for $2 \leq i \leq l-1$, $S_{1}$ has endpoints $u_{2}$ and $v_{2}$, and $S_{l}$ has endpoints $u_{l-1}$ and $v_{l-1}$. Let the paths $Q=\bigcup_{2}^{l-2} Q_{i}$ and $R=\bigcup_{2}^{l-2} R_{i}$.

For every $i \in I$, we want to define a non-zero path $\overline{P_{i}}$ extending $P_{i}$ such that the endpoints of $\overline{P_{i}}$ are contained in $R \cup Q$. Towards this end, for every $i \in I$, let $x_{i}$ and $y_{i}$ be the endpoints of $P_{i}$. If $x_{i}, y_{i} \in R \cup Q$, let $\bar{P}_{i}=P_{i}$. Lest $G$ contain $k$ disjoint non-zero thetas, there exists a set of at most $3 k$ indices $j, 2 \leq j \leq l-1$, such that $S_{j}$ is a non-zero path. Let $J \subseteq\{2,3, \ldots, l-1\}$ such that $S_{j}$ has weight zero for all $j \in J$. We have excluded 1 and $l$ from the set $J$ to ensure that the paths $\overline{P_{i}}$ that we construct will be pairwise disjoint. If at least one endpoint of $P_{i}$ is contained in a path $S_{j}$ for some $j \in J$, then we construct $\overline{P_{i}}$ as follows. Without loss of generality, assume $x_{i}$ is contained in $S_{j}$ for some $j \in J$. Since $\gamma\left(S_{j}\right)=0$, one of the paths $u_{j} S_{j} x_{i} P_{i} y_{i}$ or $v_{j} S_{j} x_{i} P_{i} y_{i}$ has non-zero weight. To see this, if both have weight zero, then $\gamma\left(u_{j} S_{j} x_{i}\right)=\gamma\left(v_{j} S_{j} x_{i}\right)=-\gamma\left(P_{i}\right)$. But given that $\Gamma$ does not contain elements of order two, this implies that $\gamma\left(S_{j}\right) \neq 0$ and contradicts our assumption that $j \in J$. If $y_{i} \in R \cup Q$, we let let $\overline{P_{i}}=u_{j} S_{j} x_{i} P_{i} y_{i}$ if $\gamma\left(u_{j} S_{j} x_{i} P_{i} y_{i}\right) \neq 0$ and otherwise, let $\overline{P_{i}}=v_{j} S_{j} x_{i} P_{i} y_{i}$. If $y_{i}$ is contained in $S_{j^{\prime}}$ for some $j^{\prime} \in J$, then by the same argument as above, at least one of the four paths $u_{j} S_{j} x_{i} P_{i} y_{i} S_{j^{\prime}} u_{j^{\prime}}, u_{j} S_{j} x_{i} P_{i} y_{i} S_{j^{\prime}} v_{j^{\prime}}, v_{j} S_{j} x_{i} P_{i} y_{i} S_{j^{\prime}} u_{j^{\prime}}$, or $v_{j} S_{j} x_{i} P_{i} y_{i} S_{j^{\prime}} v_{j^{\prime}}$ is a non-zero path. We set $\overline{P_{i}}$ to such a non-zero path, arbitrarily choosing one if there is more than one such non-zero path. Let $I_{1} \subseteq I$ be the set of indices for which $\overline{P_{i}}$ is defined. By construction, $\left|I_{1}\right| \geq|I|-(3 k+2) \geq 3\left(9 k^{2}\right)^{2}$. Also, by construction, $\overline{P_{i}}$ and $\overline{P_{j}}$ are disjoint for every distinct $i, j \in I_{1}$.

By Lemma 4.2, the path $\overline{P_{i}}$ has one endpoint in $Q$ and one endpoint in $R$ for at most $9 k^{2}$ distinct indices $i \in I_{1}$. Thus without loss of generality, we may assume that there exists a set $I_{2} \subseteq I_{1}$ with $\left|I_{2}\right| \geq\left(\left|I_{1}\right|-9 k^{2}\right) / 2 \geq\left(9 k^{2}\right)^{2}$ such that for every index $i \in I_{2}$, the path $\overline{P_{i}}$ has both endpoints in $R$. Let $\overline{x_{i}}$ and $\overline{y_{i}}$ be the endpoints of $\overline{P_{i}}$ for all $i \in I_{2}$. Consider the subpaths of $\overline{x_{i}} R \overline{y_{i}}$ for all $i \in I_{2}$. By Lemma 4.4, either there exists an edge of $R$ intersecting at least $9 k^{2}$ of the subpaths $\overline{x_{i}} R \overline{y_{i}}$ or there exists $9 k^{2}$ disjoint such subpaths. In the first case, say the edge $e$ intersects at least $9 k^{2}$ of the subpaths $\overline{x_{i}} R \overline{y_{i}}$. There exist $9 k^{2}$ disjoint non-zero paths, each with one endpoint in each of two paths of $R-e$. Lemma 4.2 then contradicts our choice of $G$ to not contain $k$ vertex disjoint non-zero cycles. 
In the other possible outcome of the application of Lemma 4.4, there exist $9 k^{2}$ distinct indices $i \in I_{2}$ such that the subpaths $\overline{x_{i}} R \overline{y_{i}}$ are pairwise disjoint. In fact, we will only need $2 k$ such paths to derive a contradiction. For each such $i$, without loss of generality, assume that the vertices $v_{2}, \overline{x_{i}}$, $\overline{y_{i}}, v_{l-1}$ occur on the path $R$ in that order. Let $\pi_{L}(i)$ be the index in $\{2, \ldots, l-1\}$ such that $v_{\pi_{L}(i)}$ is as close to $\overline{x_{i}}$ as possible on the subpath $v_{2} R \overline{x_{i}}$. Similarly, let $\pi_{R}(i)$ be the index such that $v_{\pi_{R}(i)}$ is as close as possible to $\overline{y_{i}}$ on the subpath $\overline{y_{i}} R v_{l-1}$. Note that $v_{\pi_{L}(i)}$ may in fact be equal to $\overline{x_{i}}$, and, symmetrically, $v_{\pi_{R}(i)}$ may be equal to $\overline{y_{i}}$. Given $2 k$ indices such that the subpaths $\overline{x_{i}} R \overline{y_{i}}$ are pairwise disjoint, there exists a subset $I_{3} \subseteq I_{2}$ of $k$ indices $i$ such that $v_{\pi_{R}(i)} \neq v_{\pi_{L}(j)}$ for all distinct $i, j \in I_{3}$. Equivalently, we see that the subpath $v_{\pi_{L}(i)} R v_{\pi_{R}(i)}$ is disjoint from $v_{\pi_{L}(j)} R v_{\pi_{R}(j)}$ for all $i \neq j$. For all $i \in I_{3}$, by the construction of the path $\overline{P_{i}}$, the path $P_{i}$ has both endpoints contained in the cycle $C_{i}:=v_{\pi_{L}(i)} S_{\pi_{L}(i)} u_{\pi_{L}(i)} Q u_{\pi_{R}(i)} S_{\pi_{R}(i)} v_{\pi_{R}(i)} R v_{\pi_{L}(i)}$. We conclude that $P_{i} \cup C_{i}$ forms a non-zero theta for each $i \in I_{3}$, and consequently, $G$ contains $k$ vertex disjoint non-zero thetas. The observation then implies $G$ contains $k$ vertex disjoint non-zero cycles, contradicting our choice of $G$. This completes the proof of the lemma.

Throughout the following lemmas, we will often consider a graph $G$ isomorphic to a subdivision of $W_{l, m}$ the $l \times m$ wall. We will include the assumption that $l$ is even and $m$ is odd to ensure that the graph has minimum degree two. Every branch vertex corresponds naturally to a vertex of the wall $W_{l, m}$. We will refer to the labeling of the set $X$ of branch vertices so that

$$
\begin{aligned}
X= & \left\{v_{i, j}: 2 \leq i \leq l-1,2 \leq j \leq m-1\right\} \cup\left\{v_{1, i}: 2 \leq i \leq m-1, i \text { odd }\right\} \cup \\
& \cup\left\{v_{l, i}: 2 \leq i \leq m-1, i \text { odd }\right\}
\end{aligned}
$$

as the canonical labeling of the branch vertices $X$. Let $P$ be a segment of $G$ with end points $v_{i, j}$ and $v_{i^{\prime}, j^{\prime}}$ for some $i, i^{\prime}, j, j^{\prime}$. If $i=i^{\prime}$, we say $P$ is a horizontal segment and otherwise say $P$ is a vertical segment.

Lemma 4.6 Let $G$ be a $\Gamma$-labeled graph with weight function $\gamma$. Assume $G$ is isomorphic to a subdivision of $W_{l, m}$ with $l$ even and $m$ odd. Also assume that $\Gamma$ does not contain any elements of order two. If $G$ has at least $24 k^{2}$ non-zero segments, then $G$ contains $k$ disjoint non-zero cycles.

Proof. Let $G, \Gamma$, and $\gamma$ be as in the statement. Let $X$ be the set of branch vertices and have the canonical labeling. Let the path $S_{i}, 1 \leq i \leq l$, be the union of every horizontal segment with end points $v_{i, j}$ and $v_{i, j+1}$ for some index $j$.

First, we will see that the lemma holds if $G$ has at least $12 k^{2}$ vertical non-zero segments. If there exists an index $i$ such that there exist $3 k$ non-zero vertical segments with one endpoint in each of the paths $S_{i}$ and $S_{i+1}$, then $G$ contains $k$ vertex disjoint non-zero thetas. Observation 1 implies that $G$ then contains $k$ vertex disjoint non-zero cycles. Notice that for any index $i, 1 \leq i \leq l-1$, any non-zero vertical segment with endpoints in $S_{i}$ and $S_{i+1}$ is contained in a non-zero theta using vertices of $S_{i-1}, S_{i}, S_{i+1}$, and $S_{i+2}$ as well as vertical segments with their endpoints contained in $S_{i-1}, S_{i}, S_{i+1}$, and $S_{i+2}$. Note that the paths $S_{i-1}$ and $S_{i+2}$ may be necessary for to find a non-zero theta containing the first or last vertical segment on the outside boundary of $H$. It follows that if there exist $4 k$ distinct indices $i$ with $1 \leq i \leq l-1$, such that there exists a non-zero vertical segment attaching to $S_{i}$ and $S_{i+1}$, then $G$ contains $k$ vertex disjoint non-zero thetas. We conclude that if there exist at least $(3 k)(4 k)=12 k^{2}$ non-zero vertical segments, then the conclusion of the lemma is satisfied. 
We will now show that if $G$ has at least $12 k^{2}$ non-zero horizontal segments, then the lemma holds as well. For any index $i, 1 \leq i \leq l$, we can naturally order the horizontal segments on $S_{i}$ by the order in which they occur when traversing the path $S_{i}$. Any non-zero horizontal segment is contained in a non-zero theta that intersects $S_{i}$ in at most the previous and the next segments on $S_{i}$. By choosing such non-zero thetas to contain as few vertices as possible, it follows that if $S_{i}$ contains at least $4 k$ non-zero segments, then $G$ contains $k$ disjoint non-zero thetas and the lemma holds. For all $i$, $2 \leq i \leq l-1$, every non-zero horizontal segment on $S_{i}$ is contained in a non-zero theta using only vertices of $S_{i-1}, S_{i}$, and $S_{i+1}$ as well as vertical segments attaching to $S_{i-1}, S_{i}$, and $S_{i+1}$. Similarly, when $i \in\{1, l\}$ and there exists a non-zero segment on $S_{i}$ then there exists a non-zero theta contained in $S_{1}, S_{2}, S_{3}$ or $S_{l}, S_{l-1}, S_{l-2}$ as well as the vertical segments attaching to them. We conclude that if there exist at least $3 k$ distinct indices such that $S_{i}$ contains a non-zero horizontal segment, then $G$ contains $k$ vertex disjoint non-zero cycles. It follows that if $G$ contains at least $(3 k)(4 k) \leq 12 k^{2}$ non-zero horizontal segments, then $G$ contains $k$ disjoint non-zero cycles.

It follows that if $G$ contains at least $24 k^{2}$ non-zero segments, then $G$ contains $k$ disjoint non-zero cycles and the lemma is proven.

Lemma 4.7 Let $G$ be a $\Gamma$-labeled graph with weight function $\gamma$. Let $l$ and $m$ be integers, $l \geq 4$, $m \geq 5$, with $l$ even and $m$ odd. Let $H$ be a subgraph of $G$ isomorphic to a subdivision of $W_{l, m}$. Assume that $\Gamma$ does not contain any elements of order two. Let $k$ and $n$ be integers with

$$
n \geq 9,320,850 k^{8} \geq 6\left(54 k^{2}+2\right)\left(27816 k^{6}+(4 k+2)\left(81 k^{4}\right)+24 k^{2}\right) .
$$

Let $P_{1}, \ldots, P_{n}$ be disjoint non-zero $V(H)$-paths in $G$ such that for all $1 \leq i \leq n$, the path $P_{i}$ does not have both endpoints contained in a segment of $H$. Then $G$ contains $k$ disjoint non-zero cycles.

Proof. Let $G, H, \Gamma, \gamma$, and $P_{1}, \ldots, P_{n}$ be as in the statement. Let $X$ be the branch vertices of $H$, and let $X$ have the canonical labeling.

We first observe that if there exists a segment $P$ of $H$ and at least $54 k^{2}+2$ distinct indices $i$ such that $P_{i}$ has an endpoint in $P$, then $G$ contains $k$ disjoint non-zero cycles. Observe that there exists a path in $H$ containing every horizontal segment. The vertical segments can be covered by two (not necessarily disjoint) paths. If we let $Y$ be the internal vertices of $P$, we see that there exists six paths covering the vertices of $H-Y$. It follows that there exists a subpath $Q$ in $H$ and at least $9 k^{2}$ distinct indices $i$ such that $P_{i}$ has one endpoint on $P$ and one endpoint in $Q$. By Lemma 4.2, $G$ contains $k$ disjoint non-zero cycles.

For every index $i$, the path $P_{i}$ has an endpoint in at most six distinct segments of $H$, three at each endpoint. We conclude that we may assume there exists a set $I \subseteq\{1,2, \ldots, n\}$ of at least $n / 6\left(54 k^{2}+2\right) \geq 27816 k^{6}+(4 k+2)\left(81 k^{4}\right)+24 k^{2}$ distinct indices such that $P_{i}$ and $P_{j}$ do not have endpoints contained in a common segment of $H$ for all distinct $i, j \in I$. For all $i, 2 \leq i \leq l-1$, we let $S_{i}$ be the union of every horizontal segment with endpoints equal to $v_{i, j}$ and $v_{i, j+1}$ for some index $j$. We let $S_{1}$ be formed from the "upper left" vertical segment with endpoints $v_{2,2}$ and $v_{1,3}$, the "upper right" vertical segment with endpoints $v_{1, m-2}$ and $v_{2, m-1}$, and every horizontal segment with endpoints $v_{1, j}$ and $v_{1, j+2}$ for some index $j$. Symmetrically, we let $S_{l}$ be the union of the "lower left" vertical segment with endpoints $v_{l-1,2}$ and $v_{l, 3}$, the "lower right" vertical segment with endpoints $v_{l-1, m-1}$ and $v_{l, m-2}$, and every horizontal segment with endpoints $v_{l, j}$ and $v_{l, j+2}$. We let $Q$ be the union of the vertical segments with endpoints $v_{i, 2}$ and $v_{i+1,2}$ for some index $i, 2 \leq i \leq l-2$. 
Symmetrically, we let $R$ be the union of the vertical segments with endpoints $v_{i, m-1}$ and $v_{i+1, m-1}$ for $i, 2 \leq i \leq l-2$.

We would like to convert the set of disjoint $V(H)$-paths $\left\{P_{i}: i \in I\right\}$ to a set of disjoint non-zero paths $\overline{P_{i}}$ attaching to the subdivided ladder on the vertex set $Z:=V(Q) \cup V(R) \cup\left(\bigcup_{1}^{l} V\left(S_{i}\right)\right)$. If $P_{i}$ has both endpoints in $Z$, then let $\overline{P_{i}}=P_{i}$. For paths $P_{i}$ such that exactly one endpoint of $P_{i}$ is contained in a vertical segment $T$ of $H$ of weight zero with $T \nsubseteq Z$, observe that $T$ has endpoints $x$ and $y$ in $Z$. Let $z$ be the endpoint of $P_{i}$ in $Z$ and $z^{\prime}$ be the endpoint in $T$. Given that the segment $T$ was assumed to have zero weight and the fact that $\Gamma$ does not contain an element of order two, either the path $z P_{i} z^{\prime} T x$ or $z P_{i} z^{\prime} T y$ is a non-zero $Z$-path. We let $\overline{P_{i}}=z P_{i} z^{\prime} T x$ if $\gamma\left(z P_{i} z^{\prime} T x\right) \neq 0$ and set $\overline{P_{i}}=z P_{i} z^{\prime} T y$ otherwise. For $i \in I$ such that the endpoints of $P_{i}$ are contained in exactly two vertical segments $T_{1}$ and $T_{2}$ with $\gamma\left(T_{1}\right)=\gamma\left(T_{2}\right)=0$ and $T_{1}, T_{2} \nsubseteq Z$, we let the endpoints of $T_{1}$ be $x_{1}$ and $y_{1}$, and we let the endpoints of $T_{2}$ be $x_{2}$ and $y_{2}$. At least one of the paths $x_{1} T_{1} z P_{i} z^{\prime} T_{2} x_{2}$, $x_{1} T_{1} z P_{i} z^{\prime} T_{2} y_{2}, y_{1} T_{1} z P_{i} z^{\prime} T_{2} x_{2}$, or $y_{1} T_{1} z P_{i} z^{\prime} T_{2} y_{2}$ must be a non-zero path. We choose one such non-zero path arbitrarily and assign it to be $\overline{P_{i}}$.

By Lemma 4.6, we may assume $H$ has at most $24 k^{2}$ non-zero segments. We conclude that there exists a set $I_{1} \subseteq I$ such that $\overline{P_{i}}$ is defined for all $i \in I_{1}$ with $\left|I_{1}\right| \geq|I|-24 k^{2} \geq 27816 k^{6}+(4 k+2)\left(81 k^{4}\right)$. Observe that for all distinct $i, j \in I_{1}, V\left(\overline{P_{i}}\right) \cap V\left(\overline{P_{j}}\right)=\emptyset$ by construction.

We would like to apply Lemma 4.5. In order to do so, we need to find a large subset of $I_{2} \subseteq I_{1}$ such that for any index $i \in I_{2}$, there does not exist an index $j, 1 \leq j \leq l$, such that the path $\frac{1}{P_{i}}$ has both endpoints contained in $S_{j}$. This will suffice to ensure that no path $\bar{P}_{i}$ has both endpoints in a single segment of the subdivided ladder on the vertex set $Z$, since by the construction no path $\bar{P}_{i}$ has both endpoints contained in a segment in $Q$ or $R$. Thus if $\bar{P}_{i}$ has both endpoints contained in a single segment of the subdivided ladder, that segment must be equal to $S_{j}$ for some index $j$ by construction.

Assume there exists an index $j, 1 \leq j \leq l$, and a subset $I^{\prime} \subseteq I_{1}$ such that $\left|I^{\prime}\right| \geq\left(9 k^{2}\right)^{2}$ and the path $\overline{P_{i}}$ has both endpoints in $S_{j}$ for all $i \in I^{\prime}$. Let the endpoints of $\overline{P_{i}}$ be $x_{i}$ and $y_{i}$. By Lemma 4.4, either there exists an edge $e$ on $S_{j}$ such that at least $9 k^{2}$ distinct $\overline{P_{i}}$ have one endpoint in each component of $S_{j}-e$, or there exists $9 k^{2}$ disjoint subpaths of the form $x_{i} \overline{P_{i}} y_{i}$. In the first case, Lemma 4.2 implies $G$ would contain $k$ disjoint non-zero cycles. Thus we may assume that there exist $9 k^{2}$ disjoint subpaths of the form $x_{i} \bar{P}_{i} y_{i}$. We will in fact only need $6 k$ such paths to construct $k$ disjoint non-zero cycles. Without loss of generality, we assume that $\overline{P_{1}}, \overline{P_{2}}, \ldots, \overline{P_{6 k}}$ are such that $x_{1}, y_{1}, x_{2}, y_{2}, \ldots, x_{6 k}, y_{6 k}$ occur on $S_{j}$ in that order. If $j \neq l$, for all $i, 1 \leq i \leq k$, there exists two vertical segments linking $S_{j}$ to $S_{j+1}$ with endpoints contained in the subpath of $S_{j}$ linking $y_{6 i}$ and $x_{6(i+1)}$. Moreover, these vertical segments can be chosen to be disjoint from the subpath $x_{6 i+3} S_{j} y_{6 i+3}$. If $j=l$, we find two such vertical segments linking to $S_{l-1}$. It follows that $G$ contains $k$ vertex disjoint non-zero theta subgraphs, and consequently, $k$ vertex disjoint non-zero cycles.

We may assume, then, that for every index $j$, there exist at most $\left(9 k^{2}\right)^{2}$ distinct indices $i$ such $\overline{P_{i}}$ has both endpoints contained in $S_{j}$. We now show that we may also assume that at most $4 k+2$ distinct indices $j$ such that there exists an index $i$ where $S_{j}$ contains both endpoints of the path $\overline{P_{i}}$. Let $J \subseteq\{2, \ldots, l-1\}$ be a subset of at least $4 k$ indices such that for every $j \in J$, the path $S_{j}$ contains both endpoints of $\overline{P_{i}}$ for some index $i$. Fix $j \in J$ and $i \in I_{1}$ such that $\overline{P_{i}}$ has both endpoints in $S_{j}$. Let the endpoints of $\overline{P_{i}}$ be $x_{i}$ and $y_{i}$, and let $W$ be the set of internal vertices of the subpath $x_{i} S_{j} y_{i}$. Given that $\Gamma$ does not contain an element of order two, either $\overline{P_{i}} \cup x_{i} S_{j} y_{i}$ forms a non-zero cycle, or one of the paths $S_{j}$ and $\left(S_{j}-W\right) \cup \overline{P_{i}}$ forms a non-zero path. Given that $|J| \geq 4 k$, we either find $k$ vertex disjoint non-zero cycles, or $3 k$ disjoint non-zero paths from $Q$ to $R$ containing $k$ 
disjoint non-zero thetas. Either case implies that $G$ contains $k$ disjoint non-zero cycles.

We conclude that there exists a set $I_{2} \subseteq I_{1}$ with $\left|I_{2}\right| \geq\left|I_{1}\right|-(4 k+2)\left(81 k^{4}\right) \geq 27816 k^{6}$ such that for every $i \in I_{2}$ and for every $j, 1 \leq j \leq l$, the path $\overline{P_{i}}$ has at most one endpoint in $S_{j}$. By the construction of the $\overline{P_{i}}$, it follows that each $\overline{P_{i}}$ has endpoints in distinct segments of the graph

formed by $Q \cup R \cup\left(\bigcup_{1}^{l} S_{i}\right)$. Lemma 4.5 implies that $G$ contains $k$ vertex disjoint non-zero cycles, completing the proof of the lemma.

\section{$5 \quad$ Proof of Theorem 3.1}

In this section, we give the proof of Theorem 3.1. We will prove the theorem for the bound $10^{86} k^{87}$ for the value of $n_{k}$. These constants are certainly far from optimal. The choice of constants was determined by two goals. First, we wanted to obtain a bound for $n_{k}$ that is a polynomial in $k$, and, second, we attempt to make the proof as simple as possible while still keeping such a polynomial bound.

We begin by formalizing what we mean by a minimal counter-example to Theorem 3.1.

Definition Let $k$ be a positive integer. We say the pair $\left(H,\left\{P_{i}: 1 \leq i \leq n\right\}\right)$ is a $k$-counter-example if the following conditions hold.

1. The integer $n$ satisfies $n \geq 10^{86} k^{87}$.

2. There exists an integer $l \geq n$ congruent to $0 \bmod 4$ and an integer $m \geq n$ congruent to $7 \bmod$ 8 , such that $H$ is isomorphic to a subdivision of the $l \times m$ wall.

3. The paths $P_{1}, \ldots, P_{n}$ are vertex disjoint $X$-paths where $X$ is the set of branch vertices of $H$.

4. There exists a group $\Gamma$ which does not have any elements of order two and a $\Gamma$-labeling of $G:=H \cup\left(\bigcup_{1}^{n} P_{i}\right)$ such that each $P_{i}$ is a non-zero path and the graph $G$ does not contain $k$ vertex disjoint non-zero cycles. Such a labeling $\gamma$ will be referred to as a testifying $\Gamma$-labeling.

The pair $\left(H,\left\{P_{i}: 1 \leq i \leq n\right\}\right)$ is $k$-minimal if it is a $k$-counter-example and there does not exist a $k$-counter-example containing fewer edges.

The proof of Theorem 3.1 will proceed by showing that no such $k$-minimal pair $\left(H,\left\{P_{i}: 1 \leq i \leq\right.\right.$ $n\}$ ) exists. If the paths $P_{1}, \ldots, P_{n}$ were disjoint from $H$ except for their endpoints, then the proof of Theorem 3.1 would follow from a relatively straight forward application of Lemma 4.6 and Lemma 4.7. The majority of the proof will be concerned with controlling how the individual $P_{i}$ intersect with the subgraph $H$. Towards that end, we give the following definitions. A leg of $P_{i}$ for some $i$ is a subpath of $P_{i}-E(H)$ forming a $V(H)$-path. A foot of $P_{i}$ is a component of $P_{i} \cap H$.

We begin with a series of lemmas.

Lemma 5.1 Let $\left(H,\left\{P_{i}: 1 \leq i \leq n\right\}\right)$ form a k-minimal pair. Then for all $1 \leq i \leq n$, no leg of $P_{i}$ has both endpoints contained in the same segment of $H$.

Proof. Let $P$ be a segment of $H$, and assume there exists a leg $Q$ of some path $P_{i}$ such that the endpoints $x$ and $y$ of $Q$ are both contained in $P$. We define the subgraph $H^{\prime}$ to be equal to $H-i n t(x P y) \cup Q$ when $x P y$ has length at least two, and set $H^{\prime}$ equal to $H-x y \cup Q$ otherwise. In 
either case, the subgraph $H^{\prime}$ is isomorphic to a subdivision of an $l \times m$ wall with the same branch vertices as $H$. It follows that $\left(H^{\prime},\left\{P_{i}: 1 \leq i \leq n\right\}\right)$ contradicts the fact that $\left(H,\left\{P_{i}: 1 \leq i \leq n\right\}\right)$ is $k$-minimal.

Lemma 5.2 Let $\left(H,\left\{P_{i}: 1 \leq i \leq n\right\}\right)$ form a $k$-minimal pair. Let $\gamma$ be a testifying $\Gamma$-labeling of $H \cup\left(\bigcup_{1}^{n} P_{i}\right)$. There exists a subset $I \subseteq\{1,2, \ldots, n\}$ with $|I| \geq n-10^{7} k^{8}$ such that for every $i \in I$, every leg of $P_{i}$ has weight zero in the testifying labeling.

Proof. The claim follows immediately from Lemma 4.7. If there exists a set of at least $10^{7} k^{8}$ distinct indices $i$ such that $P_{i}$ contains a non-zero leg, then since no leg has both endpoints contained in a segment of $H$, Lemma 4.7 implies that $G$ contains $k$ vertex disjoint non-zero cycles, a contradiction.

For any index $i$ such that the corresponding $P_{i}$ in a $k$-minimal pair has only legs of weight zero, there must exist some foot of $P_{i}$ that has non-zero weight.

Lemma 5.3 Let $\left(H,\left\{P_{i}: 1 \leq i \leq n\right\}\right)$ be a k-minimal pair with testifying $\Gamma$-labeling $\gamma$. Let $I$ be the indices such that $P_{i}$ has no non-zero leg for all $i \in I$. Let $P$ be a segment of $H$ and let $J \subseteq I$ be the set of indices such that $P_{i}$ has a non-zero foot on $P$. Then $|J| \leq 10^{65} k^{77}$.

Proof. Let $\left(H,\left\{P_{i}: 1 \leq i \leq n\right\}\right), I, \Gamma$ and $\gamma$ be as in the statement. Let $G=H \cup\left(\bigcup_{1}^{n} P_{i}\right)$. Fix $P$ to be a segment of $H$, and let

$$
J:=\left\{i \in I: P_{i} \text { has a non-zero foot in } P\right\} .
$$

We assume that

$$
|J| \geq 10^{65} k^{77} \geq\left[(4 k)\left[12\left(10^{7}(k+1)^{8}\right)\right]\left(192\left(9 k^{2}+1\right)^{4}\right)+4 k+2\right]^{4} 10^{8}(k+1)^{8}+10^{7}(k+1)^{8},
$$

and we will show that in this case $G$ contains $k$ vertex disjoint non-zero cycles. This contradiction implies the statement of the lemma.

The proof proceeds by repeatedly refining the set $J$. We will need the following constants.

$$
\begin{aligned}
& n_{1}=\left[(4 k)\left[12\left(10^{7}(k+1)^{8}\right)\right]\left(192\left(9 k^{2}+1\right)^{4}\right)+4 k+2\right]^{4} \\
& n_{2}=\sqrt{n_{1}} \\
& n_{3}=\sqrt{n_{2}}-k-2 \\
& n_{4}=n_{3}-3 k \\
& n_{5}=n_{4} / 192\left(9 k^{2}+1\right)^{4} \geq(4 k) 12\left(10^{7}(k+1)^{8}\right)
\end{aligned}
$$

For every $i \in J$, let $F_{i}$ be a non-zero foot of $P_{i}$ contained in the segment $P$. Let the endpoints of $F_{i}$ be $x_{i}$ and $y_{i}$ for all $i \in J$. Let $F_{i}^{\prime}$ be the union of $F_{i}$ and the two legs of $P_{i}$ intersecting $F_{i}$. Observe that for all $i \in J$, the path $F_{i}^{\prime}$ is a non-zero path with both endpoints in $V(H)-\operatorname{int}(P)$.

Claim 5.4 There exists a subset $J_{1} \subseteq J$ and a segment $Q$ of $H$ such that for all $i \in J_{1}$, every path $F_{i}^{\prime}$ has both endpoints contained in $Q$. Moreover, $\left|J_{1}\right| \geq n_{1}$. 
Proof. Lemma 4.7 implies that the path $F_{i}^{\prime}$ has endpoints in distinct segments of $H$ for at most $10^{7}(k+1)^{8}$ distinct indices. To see this, assume there exist $10^{7}(k+1)^{8}$ such indices. Let $e$ be a new edge with endpoints equal to the endpoints of $P$. The graph $(H-\operatorname{int}(P)) \cup e \cup\left\{F_{i}^{\prime}: i \in J\right\}$ would contain $k+1$ non-zero cycles by Lemma 4.7. Conseqently, $(H-\operatorname{int}(P)) \cup\left\{F_{i}^{\prime}: i \in J\right\}$ would contain $k$ disjoint non-zero cycles, a contradiction.

We now fix a subset $J^{\prime} \subseteq J$ and distinct segments $Q_{i}$ of $H$ such that for all $i \in J^{\prime}$, the path $F_{i}^{\prime}$ has both endpoints contained in $Q_{i}$, and furthermore, for all distinct $i, j \in J^{\prime}, Q_{i} \neq Q_{j}$. Assume we can choose $J^{\prime}$ such that $\left|J^{\prime}\right| \geq 10^{8}(k+1)^{8} \geq 5 \cdot 10^{7}(k+1)^{8}$. Observe that for any distinct indices $i, j \in J^{\prime}$, there exists a non-zero path $R$ with one endpoint in $Q_{i}$ and the second endpoint in $Q_{j}$ where $R \subseteq F_{i}^{\prime} \cup F_{j}^{\prime} \cup x_{i} P x_{j}$. As a slight technicality, in order to ensure that the path $R$ has endpoints on distinct segments of $H$, we require $Q_{i}$ and $Q_{j}$ to not have a common endpoint. Since every segment of $H$ has a common endpoint with at most four other segments, we conclude that there exists a subset $J^{\prime \prime} \subseteq J^{\prime}$ with $\left|J^{\prime \prime}\right| \geq\left|J^{\prime}\right| / 5$ such that for all $i, j \in J^{\prime \prime}, Q_{i}$ and $Q_{j}$ are disjoint. We conclude that there exist $10^{7}(k+1)^{8}$ disjoint non-zero paths attaching to $H-\operatorname{int}(P)$. As in the previous paragraph, $G$ contains $k$ disjoint non-zero cycles.

We conclude that there exists a segment $Q$ of $H$ such that at least $n_{1} \geq\left(|J|-10^{7}(k+1)^{8}\right) / 10^{8}(k+$ $1)^{8}$ indices $i$, the path $F_{i}^{\prime}$ has both endpoints in the segment $Q$, and the claim is proven.

For the remainder of the proof of Lemma 5.3, we fix $J_{1}$ and the segment $Q$ to be as in the statement of Claim 5.4. We fix the labels $x_{i}^{\prime}$ and $y_{i}^{\prime}$ to be the endpoints of $F_{i}^{\prime}$ in $Q$.

Claim 5.5 There exists a subset $J_{2} \subseteq J_{1}$ with $\left|J_{2}\right| \geq n_{2}$ such that for all distinct $i, j \in J_{2}$, the subpaths $x_{j}^{\prime} Q y_{j}^{\prime}$ and $x_{i}^{\prime} Q y_{i}^{\prime}$ are disjoint.

Proof. We apply Lemma 4.4 to either find an edge $e$ in $Q$ intersecting at least $n_{2} \geq 9 k^{2}$ of the paths $x_{i}^{\prime} Q y_{i}^{\prime}$, or a subset of $n_{2}$ distinct indices $i$ such that the paths $x_{i}^{\prime} Q y_{i}^{\prime}$ are pairwise disjoint. In the first case, there exist $9 k^{2}$ disjoint non-zero paths attaching to the two components of $Q-e$. Lemma 4.2 implies that $G$ would then contain $k$ vertex disjoint non-zero cycles. We conclude that there exists a subset $J_{2} \subseteq J_{1}$ with $\left|J_{2}\right| \geq \sqrt{\left|J_{1}\right|} \geq n_{2}$ such that the subpaths $x_{i}^{\prime} Q y_{i}^{\prime}$ are pairwise disjoint for all $i \in J_{2}$.

Let the endpoints of $P$ be $u_{1}$ and $v_{1}$ and let the endpoints of $Q$ be $u_{2}$ and $v_{2}$. There is a natural ordering, denoted by $\preceq_{P}$, of the vertices of $P$ where for any two vertices $z, z^{\prime}$ on $P, z \preceq_{P} z^{\prime}$ if $z$ is closer to $u_{1}$ on the path $P$. Similarly, for any two vertices of $z$ and $z^{\prime}$ on $Q, z \preceq_{Q} z^{\prime}$ if $z$ is closer to $u_{2}$ on $Q$. In a slight abuse of notation, we will use $\preceq_{P}$ to order the feet laying on $P$ where $F_{i} \preceq_{P} F_{j}$ if $F_{i}$ is closer to the vertex $u_{1}$ on the path $P$. Similarly, we use $\preceq_{Q}$ to order the subpaths $x_{i}^{\prime} Q y_{i}^{\prime}$ on $Q$.

Claim 5.6 By possibly swapping the labels $v_{1}$ and $u_{1}$ on $P$, there exists a set $J_{3} \subseteq J_{2}$ such that $\left|J_{3}\right|=n_{3}$ and the following hold:

i. for all $i, j \in J_{3}$, if $F_{i} \preceq_{P} F_{j}$, then $x_{i}^{\prime} Q y_{i}^{\prime} \preceq_{Q} x_{j}^{\prime} Q y_{j}^{\prime}$,

ii. for all $i \in J_{3}$, the subpath $F_{i}$ does not intersect the endpoints of $P$ and similarly, the subpath $x_{i}^{\prime} Q y_{i}^{\prime}$ does not intersect the endpoints of $Q$, and

iii. for all $i \in J_{3}, \gamma\left(x_{i}^{\prime} Q y_{i}^{\prime}\right)=-\gamma\left(F_{i}\right)$. 
Proof. By the same argument as in the proof of Lemma 4.2, there exists a subset $J^{\prime} \subseteq J_{2}$ with $\left|J^{\prime}\right| \geq\left\lfloor\sqrt{\left|J_{2}\right|}\right\rfloor$ such that one of the following holds. For all $i, j \in J^{\prime}$, if $F_{i} \preceq_{P} F_{j}$, then $x_{i}^{\prime} Q y_{i}^{\prime} \preceq_{Q} x_{j}^{\prime} Q y_{j}^{\prime}$, or, for all $i, j \in J^{\prime}$, if $F_{i} \preceq_{P} F_{j}$, then $x_{j}^{\prime} Q y_{j}^{\prime} \preceq_{Q} x_{i}^{\prime} Q y_{i}^{\prime}$. By possibly swapping the labels $u_{1}$ and $v_{1}$ on $P$, we may assume the former holds and that $J^{\prime}$ satisfies $i$. By possibly discarding the indices corresponding to the first and last feet on $P$, we may assume that for all $i \in J^{\prime}$, the subpath $F_{i}$ does not intersect the endpoints of $P$ and similarly, the subpath $x_{i}^{\prime} Q y_{i}^{\prime}$ does not intersect the endpoints of $Q$. Thus $J^{\prime}$ satisfies $i i$. Finally, observe that if there exist $k$ distinct indices $i$ such that $\gamma\left(x_{i}^{\prime} Q y_{i}^{\prime}\right) \neq-\gamma\left(F_{i}\right)$, then $G$ would contain $k$ disjoint non-zero cycles. By discarding all such indices in $J^{\prime}$, we may assume $J^{\prime}$ satisfies iii. as well. We conclude that we have constructed the set $J_{3}$ with $\left|J_{3}\right|=\left\lfloor\sqrt{\left|J_{2}\right|}\right\rfloor-k-2=n_{3}$, and the claim is proven.

We re-number the paths $F_{i}$ with $i \in J_{3}$ so that $J_{3}=\left\{1,2, \ldots, n_{3}\right\}$. For all $i \in J_{3}$, we fix the labels $x_{i}, y_{i}, x_{i}^{\prime}, y_{i}^{\prime}$ of $F_{i}^{\prime}$ such that the vertices $x_{1}, y_{1}, x_{2}, y_{2}, \ldots, x_{n_{3}}, y_{n_{3}}$ occur on $P$ in that order when traversing from $u_{1}$ to $v_{1}$, and and the vertices $x_{1}^{\prime}, y_{1}^{\prime}, x_{2}^{\prime}, y_{2}^{\prime}, \ldots, x_{n_{3}}^{\prime}, y_{n_{3}}^{\prime}$ occur on $Q$ in that order when traversing $Q$ from $u_{2}$ to $v_{2}$. Throughout the remainder of the proof, as we continue to further refine the set $J_{3}$ we will re-number the indices to be the set $\left\{1,2, \ldots, n^{\prime}\right\}$ for some integer $n^{\prime}$. When we do so, we do so such that the paths $F_{1} \preceq_{P} F_{2} \preceq_{P} F_{3} \preceq_{P} \cdots \preceq_{P} F_{n^{\prime}}$.

Observe that for each $i \in J_{3}$ the two legs contained in $F_{i}^{\prime}$ either link $x_{i}$ to $x_{i}^{\prime}$ and link $y_{i}$ to $y_{i}^{\prime}$, or vice versa and link $x_{i}$ to $y_{i}^{\prime}$ and $y_{i}$ to $x_{i}^{\prime}$. In the latter case, we say that the path $F_{i}^{\prime}$ twists.

Claim 5.7 There are at most $3 k$ distinct indices $i \in J_{3}$ for which the path $F_{i}^{\prime}$ twists.

Proof. Let $i, i, 2 \leq i \leq\left|J_{3}\right|-1$, be a fixed index such that the path $F_{i}^{\prime}$ twists. There exists two internally disjoint paths linking the endpoints $x_{i}$ and $y_{i}$ of the non-zero foot $F_{i}$ avoiding any internal vertex of $F_{i}$. Moreover, these paths can be chosen to use only the vertices of $F_{i-1}^{\prime}$ and $F_{i+1}^{\prime}$ as well as the subpaths $x_{i-1} P y_{i+1}$ and $x_{i-1} Q y_{i+1}$. To see this, one such path linking $x_{i}$ and $y_{i}$ is formed by the leg $x_{i} F_{i}^{\prime} y_{i}^{\prime}$ and a subpath of $F_{i+1}^{\prime}$ linking $y_{i} P x_{i+1}$ and $y_{i}^{\prime} Q x_{i+1}^{\prime}$. The second path is obtained from the leg $y_{i} F_{i}^{\prime} x_{i}^{\prime}$ and a subpath of $F_{i-1}^{\prime}$ linking $y_{i-1}^{\prime} Q x_{i}^{\prime}$ and $y_{i-1} P x_{i}$. It follows that if there existed at least $3 k$ distinct indices $i \in J_{3}$ such that $F_{i}^{\prime}$ were twisted, then $G$ would contain $k$ vertex disjoint non-zero thetas, and consequently, $k$ disjoint non-zero cycles.

We conclude, by discarding all indices $i$ such that $F_{i}^{\prime}$ twists and by possibly renumbering the remaining feet $F_{i}$, that $F_{i}^{\prime}$ is not twisted for every $i \in J_{4}$ where $J_{4}=\left\{1,2, \ldots, n_{4}\right\}$.

For each $i \in J_{4}$, we now want to follow the path $P_{i}$ starting at each of the vertices $x_{i}^{\prime}$ and $y_{i}^{\prime}$ to their attachments in $H-(\operatorname{int}(P) \cup \operatorname{int}(Q))$. For all $i \in J_{4}$, let $\overline{F_{i}}$ be the subpath of $P_{i}$ that forms a $V(H-(\operatorname{int}(P) \cup \operatorname{int}(Q)))$-path containing $F_{i}$.

We will use the $\overline{F_{i}}$ to find many non-zero paths attaching to distinct segments in either $H-i n t(Q)$ or in $H-\operatorname{int}(P)$. Applying Lemma 4.7 will then imply that $G$ contains many non-zero cycles. If each $\bar{F}_{i}$ consisted of $F_{i}^{\prime}$ as well as two legs linking $x_{i}^{\prime}$ and $y_{i}^{\prime}$ to $H-(\operatorname{int}(P) \cup \operatorname{int}(Q))$, then it would be relatively easy to find $k$ disjoint non-zero cycles. The difficulty remaining in the proof of the lemma is that each $\bar{F}_{i}$ may contain arbitrarily many legs passing between $P$ and $Q$ before leaving $P \cup Q$ to terminate in $H-(\operatorname{int}(P) \cup \operatorname{int}(Q))$.

We define the subpaths $D_{i}$ and $D_{i}^{\prime}$ for $0 \leq i \leq n_{4}$ as follows. For $i$ such that $1 \leq i \leq n_{4}-1$, let $D_{i}$ be the path $x_{i} P x_{i+1}-\left\{x_{i+1}\right\}$ and let $D_{i}^{\prime}$ be the path $x_{i}^{\prime} Q x_{i+1}^{\prime}-\left\{x_{i+1}^{\prime}\right\}$. We let $D_{0}$ be the path $u_{1} P x_{1}-\left\{x_{1}\right\}$ and let $D_{0}^{\prime}$ be the path $u_{2} Q x_{1}^{\prime}-\left\{x_{1}^{\prime}\right\}$. Let $D_{n_{4}}$ be the path $x_{n_{4}} P v_{1}$ and let $D_{n_{4}}^{\prime}$ be the path $x_{n_{4}}^{\prime} Q v_{2}$. A leg $S$ of a path $\overline{F_{i}}$ for some $i, 1 \leq i \leq n_{4}$ is long if $S$ has one endpoint in $D_{j}$ and one endpoint in $D_{l}^{\prime}$ for indices $j$ and $l$ with $|l-j| \geq 2$. 
We now show that there are a bounded number of indices $i \in\left\{0,1, \ldots, n_{4}\right\}$ such that the subpath $D_{i}$ or $D_{i}^{\prime}$ contains an endpoint of a long leg.

Claim 5.8 There are at most $192\left(9 k^{2}+1\right)^{4}$ distinct indices $i$ such that $D_{i} \cup D_{i}^{\prime}$ contains the endpoint of a long leg.

Proof. To prove this, there are two distinct cases to eliminate: first, when there many distinct pairs $(a, b)$ such that there exists a long leg with one endpoint in $D_{a}$ and one endpoint in $D_{b}^{\prime}$, and, secondly, when there exists a fixed index $a$ and many distinct indices $b$ such that there exists a long leg with one endpoint in $D_{a} \cup D_{a}^{\prime}$ and one endpoint in $D_{b}^{\prime} \cup D_{b}$.

Case 1: There exist $\left(9 k^{2}+1\right)^{2}$ disjoint pairs $\{a, b\}$ such that some long leg has one end in $D_{a} \cup D_{a}^{\prime}$ and one end in $D_{b} \cup D_{b}^{\prime}$. Let $\left\{\{a(i), b(i)\}: 1 \leq i \leq\left(9 k^{2}+1\right)^{2}\right\}$ be a collection of $\left(9 k^{2}+1\right)^{2}$ such disjoint pairs. Assume the pairs are ordered such that $a(i)<b(i)$ for all $1 \leq i \leq\left(9 k^{2}+1\right)^{2}$. By applying Lemma 4.3, there exists a subset of $L \subseteq\left\{1,2, \ldots,\left(9 k^{2}+1\right)^{2}\right\}$ of distinct indices $i$ with $|L| \geq 9 k^{2}+1$ such that one of the following holds.

1. There exists an index $j$ such that for every pair $\{a(i), b(i)\}$ for $i \in L a(i)<j$ and $b(i)>j$.

2. Alternatively, for all distinct $i, i^{\prime} \in L$ either $a(i) \geq b\left(i^{\prime}\right)$ or $b(i) \leq a\left(i^{\prime}\right)$.

Assume the first possibility holds, and there exists an index $j$ such that for every $i \in L, a(i)<$ $j<b(i)$. For all $i \in L$, let $R_{i}$ be a long leg with endpoints in $D_{a(i)} \cup D_{a(i)}^{\prime} \cup D_{b(i)} \cup D_{b(i)}^{\prime}$. As a technicality, we assume that $a(i) \neq 0$ for all $i \in L$. Observe that the union of the subgraph $D_{a(i)} \cup F_{a(i)}^{\prime} \cup D_{b(i)} \cup F_{b(i)}^{\prime} \cup R_{i}$ contains a non-zero path with one end in $D_{a(i)}^{\prime}$ and one endpoint in $D_{b(i)}^{\prime}$. It follows that $G$ contains $9 k^{2}$ disjoint non-zero paths with one endpoint in each of the two components of $Q-D_{j}^{\prime}$. Lemma 4.2 contradicts our choice of $G$.

We consider the second possibility when for every distinct $i, i^{\prime} \in L$ either $a(i) \geq b\left(i^{\prime}\right)$ or $b(i) \leq$ $a\left(i^{\prime}\right)$. Equivalently, we see that for every distinct $i, i^{\prime} \in L$, the subpaths of $P$ given by $x_{a(i)} P x_{b(i)+1}-$ $\left\{x_{b(i)+1}\right\}$ and $x_{a\left(i^{\prime}\right)} P x_{b\left(i^{\prime}\right)+1}-\left\{x_{b\left(i^{\prime}\right)+1}\right\}$ are disjoint. Again, for all $i \in L$, let $R_{i}$ be a long leg with one endpoint in $D_{a(i)} \cup D_{a(i)}^{\prime}$ and the other endpoint in $D_{b(i)} \cup D_{b(i)}^{\prime}$. By the fact that we are considering long leaps, for every $i \in L$, there exists an index $c(i)$ such that $a(i)<c(i)<b(i)$. It now suffices to show that for any index $i \in L$, there exists a non-zero cycle contained in the subgraph $S_{i}:=x_{a(i)} P x_{b(i)+1}-\left\{x_{b(i)+1}\right\} \cup x_{a(i)}^{\prime} Q x_{b(i)+1}^{\prime}-\left\{x_{b(i)+1}^{\prime}\right\} \cup F_{a(i)}^{\prime} \cup F_{b(i)}^{\prime} \cup R_{i} \cup F_{c(i)}^{\prime}$. Again, as a technicality, we assume that $a(i) \neq 0$. Then such a non-zero cycle exists since there exists a path linking $x_{c(i)}$ and $y_{c(i)}$ in $S_{i}$ avoiding $F_{c(i)}^{\prime} \cup x_{c(i)}^{\prime} Q y_{c(i)}^{\prime}$ except at its endpoints by using the path $R_{i}$. Consequently, the subgraph $S_{i}$ contains a non-zero theta, and therefore, a non-zero cycle. Given that $|L| \geq k+1$, we see that $G$ contains $k$ vertex disjoint non-zero cycles. This contradiction completes the analysis of the first case.

Case 2: There exists an index $a$ and $6\left[4\left(9 k^{2}\right)+1\right]^{2}$ distinct indices $b$ such that some long leg has one end in $D_{a} \cup D_{a}^{\prime}$ and one end in $D_{b} \cup D_{b}^{\prime}$. Fix $a$ to be such an index and define the set $\left\{\{a, b(i)\}: 1 \leq i \leq 6\left[4\left(9 k^{2}\right)+1\right]^{2}\right\}$ of pairs be such that there exists a long leg with one endpoint in $D_{a} \cup D_{a}^{\prime}$ and a second endpoint in $D_{b(i)} \cup D_{b(i)}^{\prime}$. We partition the long legs into those that have an endpoint in $D_{a}$ and those that have an endpoint in $D_{a}^{\prime}$. We assume that for at least $3\left[4\left(9 k^{2}\right)+1\right]^{2}$ of the pairs $\{a, b(i)\}$ there exists a long leg with one endpoint in $D_{a}$ and one endpoint in $D_{b(i)}^{\prime}$. The 
analysis would follow similarly when $3\left[4\left(9 k^{2}\right)+1\right]^{2}$ of the pairs $\{a, b(i)\}$ correspond to a long leg with an endpoint in $D_{a}^{\prime}$. For any three such long legs with an endpoint in $D_{a}$, we can define a path $T$ with endpoints in $D_{j}^{\prime}$ and $D_{j^{\prime}}^{\prime}$ for some indices $j$ and $j^{\prime}$ with $\left|j-j^{\prime}\right| \geq 2$. Thus, we can define paths $T_{1}, T_{2}, \ldots, T_{m}$ where for all indices $i, 1 \leq i \leq m$, the path $T_{i}$ has endpoints in $D_{j}^{\prime}$ and $D_{j^{\prime}}^{\prime}$ for some $j$ and $j^{\prime}$ with $\left|j-j^{\prime}\right| \geq 2$ where $m \geq\left[4\left(9 k^{2}\right)+1\right]^{2}$. We construct the paths $T_{1}, \ldots, T_{m}$ to be internally disjoint from $Q \cup\left(P-D_{a}\right) \cup\left(\bigcup_{i \neq a} F_{i}^{\prime}\right)$. Notice, for all $1 \leq i \leq m$, the subpath of $Q$ linking the endpoints of $T_{i}$ contains the path $x_{j}^{\prime} Q y_{j}^{\prime}$ for some index $j$. Also, by construction, for all $i, j, 1 \leq i, j \leq m$, the path $T_{i}$ and $T_{j}$ do not have endpoints in a common $D_{j^{\prime}}^{\prime}$ for any index $j^{\prime}$. It follows that if there exist $k+1$ distinct paths $T_{i}$ such that the subpath of $Q$ defined by the endpoints of $T_{i}$ are pairwise disjoint, then $G$ would contain $k$ disjoint non-zero thetas, a contradiction. We would need $k+1$ such non-overlapping paths, as one such path may contain $D_{a}^{\prime}$.

We conclude from Lemma 4.3 that there exists an index $a^{\prime}$ such that at least $4\left(9 k^{2}\right)+1$ such $T_{i}$ have an endpoint in each component of $Q-D_{a^{\prime}}^{\prime}$. For every $1 \leq i \leq m$, if the path $T_{i}$ has endpoints in $D_{j}^{\prime}$ and $D_{j^{\prime}}^{\prime}$, for some indices $j$ and $j^{\prime}, j, j^{\prime} \neq m$, then there exists a non-zero path $T_{i}^{\prime}$ with endpoints in $P$ such that $T_{i}^{\prime} \subseteq T_{i} \cup D_{j}^{\prime} \cup D_{j^{\prime}}^{\prime} \cup D_{j^{\prime}+1}^{\prime} \cup F_{j}^{\prime} \cup F_{j^{\prime}+1}$. To see this, the subgraph $T_{i} \cup D_{j}^{\prime} \cup F_{j}^{\prime} \cup D_{j^{\prime}}^{\prime}$ contains a path from $P \cap D_{j}$ to the vertex $x_{j^{\prime}+1}^{\prime}$. Then the subgraph $F_{j^{\prime}+1}^{\prime} \cup x_{j^{\prime}+1}^{\prime} Q y_{j^{\prime}+1}^{\prime}$ contains two distinctly weighted paths from $x_{j^{\prime}+1}^{\prime}$ to $P \cap D_{j^{\prime}+1}$ ensuring that the desired non-zero path exists. Each such non-zero $T_{i}^{\prime}$ may intersect at most one other $T_{i^{\prime}}^{\prime}$ by construction, so we conclude that there are at least $2\left(9 k^{2}\right)$ disjoint non-zero paths having one endpoint in each of the two components of $P-e$ for some edge $e \subseteq D_{a^{\prime}}$. One component of $P-e$ contains the subpath $D_{a}$. It follows that there exist two components of $P-\left\{D_{a} \cup D_{a^{\prime}}\right\}$ and a set of $9 k^{2}$ disjoint non-zero paths with one endpoint in each component. Lemma 4.2 implies $G$ contains $k$ disjoint non-zero cycles, a contradiction.

We have completed the analysis of the two cases. In order to get a general bound on the number of distinct pairs of indices containing a long leg, we consider the following bipartite auxiliary graph. Let the vertex set be $\left\{u_{0}, u_{1}, \ldots, u_{n_{4}}, v_{0}, v_{1}, \ldots, v_{n_{4}}\right\}$ and for all indices $i, j$, two vertices $u_{i}$ and $v_{j}$ adjacent if there exists a long leg with endpoints in $D_{i}$ and $D_{j}^{\prime}$. From the first case, we see that the auxiliary graph has at most $\left(9 k^{2}+1\right)^{2}$ disjoint edges. By König's theorem on matchings in bipartite graphs, we see that the auxiliary graph has subset of the vertices of size at most $\left(9 k^{2}+1\right)^{2}$ incident every edge of the auxiliary graph. In the analysis of the second case, we saw that each vertex of the auxiliary graph has degree at most $6\left[4\left(9 k^{2}\right)+1\right]^{2}$. It follows that the auxiliary graph has at most $\left(9 k^{2}+1\right)^{2} 6\left[4\left(9 k^{2}\right)+1\right]^{2} \leq 96\left(9 k^{2}+1\right)^{4}$ edges, and, consequently, there are at most $96\left(9 k^{2}+1\right)^{4}$ distinct pairs of indices $i, j$ such that there is a long leg with one endpoint in $D_{i} \cup D_{i}^{\prime}$ and one endpoint in $D_{j} \cup D_{j}^{\prime}$. It follows that there are at most $192\left(9 k^{2}+1\right)^{4}$ indices $i$ such that $D_{i} \cup D_{i}^{\prime}$ contains an endpoint of a long leg. This completes the proof of the claim.

An immediate consequence of Claim 5.8 is that there exists an index $a \in J_{4}$ such that for all $i$, $1 \leq i \leq n_{5}=n_{4} / 192\left(9 k^{2}+1\right)^{4}$, there does not exist a long leg with an endpoint in $D_{a+i} \cup D_{a+i}^{\prime}$. We now fix such an index $a$ for the remainder of the proof of Lemma 5.3. For any index $i, 1 \leq i \leq n_{5}$, we say that $D_{a+i} \cup D_{a+i}^{\prime}$ is criss-crossed if there exist distinct indices $j, j^{\prime} \in\left\{1,2, \ldots, n_{4}\right\}-\{a+i\}$, and subpaths $E_{j}$ and $E_{j^{\prime}}$ of $\overline{F_{j}}$ and $\overline{F_{j^{\prime}}}$, respectively, such that the following holds:

1. $E_{j}$ and $E_{j^{\prime}}$ each have one endpoint in $D_{a+i+1} \cup D_{a+i+1}^{\prime}$ and one endpoint in $D_{a+i-1} \cup D_{a+i-1}^{\prime}$,

2. every leg of $E_{j}$ and $E_{j^{\prime}}$ has both endpoints in the set $D_{a+i} \cup D_{a+i}^{\prime}, D_{a+i-1} \cup D_{a+i-1}^{\prime}$, and $D_{a+i+1} \cup D_{a+i+1}^{\prime}$, and 
3. there do not exist subpaths of $E_{j}$ and $E_{j^{\prime}}$ satisfying 1 . and 2.

We now see that there are at most a bounded number of indices $i$ where $D_{a+i} \cup D_{a+i}^{\prime}$ is crisscrossed.

Claim 5.9 There are at most $4 k$ distinct indices $i, 1 \leq i \leq n_{5}$ such that $D_{a+i} \cup D_{a+i}^{\prime}$ is criss-crossed.

Proof. Let $i$ be an index such that $D_{a+i} \cup D_{a+i}^{\prime}$ is criss-crossed. Let $j$ and $j^{\prime}$ be indices and $E_{j}$ and $E_{j^{\prime}}$ be the two paths as in the definition of criss-crossed. We define the subgraph $H^{\prime}$ to be the subgraph formed by $x_{a+i-1} P x_{a+i+2} \cup x_{a+i-1}^{\prime} Q x_{a+i+2}^{\prime} \cup F_{a+i-1}^{\prime} \cup F_{a+i}^{\prime} \cup F_{a+i+1}^{\prime} \cup F_{a+i+2}^{\prime} \cup E_{j} \cup E_{j^{\prime}}$ To prove the claim, it suffices to show that $H^{\prime}$ contains a non-zero cycle. We do this by showing that $H^{\prime}$ contains a non-zero theta. Define the cycles $C$ and $C^{\prime}$ such that $C=x_{a+i-1} F_{a+i-1}^{\prime} x_{a+i-1}^{\prime} \cup x_{a+i-1}^{\prime} Q x_{a+i}^{\prime} \cup$ $x_{a+i}^{\prime} F_{a+i}^{\prime} x_{a+i} \cup x_{a+i} P x_{a+i-1}$ and $C^{\prime}=y_{a+i} F_{a+i}^{\prime} y_{a+i}^{\prime} \cup y_{a+i}^{\prime} Q x_{a+i+2}^{\prime} \cup x_{a+i+2}^{\prime} F_{a+i}^{\prime} x_{a+i+2} \cup x_{a+i+2} P y_{a+i}$. Observe that $D_{a+i-1} \cup D_{a+i-1}^{\prime} \subseteq V(C)$ and $D_{a+i+1} \cup D_{a+i+1}^{\prime} \subseteq V\left(C^{\prime}\right)$. Consequently, there exists two vertex disjoint subpaths of $E_{j}$ and $E_{j^{\prime}}$ each with one endpoint in $C$ and one endpoint in $C^{\prime}$. It follows that $H^{\prime}$ contains two internally disjoint paths linking $x_{a+i}$ and $y_{a+i}$ avoiding the non-zero path $F_{a+i}$. We conclude that $H^{\prime}$ contains a non-zero theta, and the claim is proven.

Given that $n_{5} \geq(4 k) 12\left(10^{7}(k+1)^{8}\right)$, an immediate consequence of Claim 5.9 is that there exists an index $b \in\left\{a+1, a+2, \ldots, a+n_{5}\right\}$ such that for all $1 \leq i \leq 12\left(10^{7}(k+1)^{8}\right), D_{b+i} \cup D_{b+i}^{\prime}$ is not crisscrossed. We are now able to complete the lemma. For $i, 4 \leq i \leq 12\left(10^{7}(k+1)^{8}\right)-3$, one of the paths $\bar{F}_{b+i-1}, \bar{F}_{b+i}$, or $\bar{F}_{b+i+1}$ must contain a leg with one endpoint in the set $\bigcup_{i-2 \leq i^{\prime} \leq i+2}\left(D_{b+i^{\prime}} \cup D_{b+i^{\prime}}^{\prime}\right)$ and one endpoint in $H-(P \cup Q)$. This follows from our choice of $b$ to avoid indices that are crisscrossed and long leaps. If none of the paths $\bar{F}_{b+i-1}, \bar{F}_{b+i}$, nor $\bar{F}_{b+i+1}$ has such a leg, then each must intersect either the set $D_{b+i-3} \cup D_{b+i-3}^{\prime}$ or the set $D_{b+i+3} \cup D_{b+i+3}^{\prime}$ since there is no long leg attaching to any of the sets $D_{b+i-2} \cup D_{b+i-2}^{\prime}, \ldots, D_{b+i+2} \cup D_{b+i+2}^{\prime}$. It follows that either the set $D_{b+i+2} \cup D_{b+i+2}^{\prime}$ or the set $D_{b+i-2} \cup D_{b+i-2}^{\prime}$ is criss-crossed, a contradiction.

We conclude that there exist $2\left(10^{7}(k+1)^{8}\right)$ distinct legs $L_{1}, L_{2}, \ldots, L_{2\left(10^{7}(k+1)^{8}\right)}$ with one endpoint in $P \cup Q$ and one endpoint in $H-(P \cup Q)$. Moreover, if we let the endpoint of $L_{i}$ in $P \cup Q$ be contained in $D_{\pi(i)} \cup D_{\pi(i)}^{\prime}$ for some $\pi(i)$, then for all distinct $i$ and $i^{\prime}$, the sets $D_{\pi(i)} \cup D_{\pi(i)}^{\prime}$ and $D_{\pi\left(i^{\prime}\right)} \cup D_{\pi\left(i^{\prime}\right)}^{\prime}$ are disjoint by construction. For each $i, 1 \leq i \leq 2\left(10^{7}(k+1)^{8}\right)$, consider the subgraph $L_{i} \cup D_{\pi(i)} \cup D_{\pi(i)}^{\prime} \cup F_{\pi(i)^{\prime}}^{\prime}$. If $L_{i}$ has one endpoint in $P$, then the subgraph $L_{i} \cup D_{\pi(i)} \cup D_{\pi(i)}^{\prime} \cup F_{\pi(i)^{\prime}}^{\prime}$ contains a non-zero path with one endpoint in $Q$ and one endpoint in $H-(P \cup Q)$ and otherwise disjoint from $Q$. Symmetrically, if $L_{i}$ has one endpoint in $Q$, there exists a non-zero path with one endpoint in $P$ and one endpoint in $H-(P \cup Q)$ and otherwise disjoint from $P$. We conclude, without loss of generality, that there exist $10^{7}(k+1)^{8}$ disjoint non-zero paths, each with one endpoint in $P$ and one endpoint in $H-(P \cup Q)$ and otherwise disjoint from $H-(P \cup Q)$. Lemma 4.7 implies that $G$ contains $k$ disjoint non-zero cycles, a contradiction. This completes the proof of Lemma 5.3.

One final step remains before we give the proof of Theorem 3.1. Consider a $k$-minimal pair $\left(H,\left\{P_{1}, \ldots, P_{n}\right\}\right)$. Let $G=H \cup\left(\bigcup_{1}^{n} P_{i}\right)$, and let $\gamma$ be a testifying $\Gamma$-labeling of $G$. Lemma 5.3 implies that there exist many disjoint segments containing a non-zero foot of some path $P_{i}$. For each such index, we will define a "breaking path". The final step of the proof of Theorem 3.1 will be to show that many breaking paths imply the existence of $k$ disjoint non-zero cycles.

A segment $S$ of $H$ with endpoints $x$ and $y$ is substantially split by a vertex $z, z \neq x, y$, if the paths $x S z$ and $y S z$ have distinct weights, i.e. $\gamma(x S z) \neq \gamma(y S z)$. A breaking path is a $V(H)$-path $Q$ 
with endpoints in distinct segments $S_{1}$ and $S_{2}$ of $H$ such that at least one of the segments $S_{1}$ or $S_{2}$ is substantially split by an endpoint of $Q$. A splitting vertex of $P_{i}^{\prime}$ is an endpoint $P_{i}^{\prime}$ that substantially splits a segment of $H$.

Consider a segment $S$ of $H$ containing a non-zero foot of some path $P_{i}$. If $\gamma(S)=0$, then one of the legs attaching to such a foot must be a breaking path. Thus Lemma $5.3 \mathrm{implies}$ that a $k$ minimal pair must contain many breaking paths such that their respective splitting vertices will lay on distinct segments of $H$. The final lemmas in this section will show how to convert such breaking paths into many disjoint non-zero cycles.

Lemma 5.10 Let $H$ be isomorphic to a subdivision of the $l \times m$ grid with $l \geq 8$ congruent to 0 mod 4 and $m \geq 23$ congruent to $7 \bmod 8$. Let $\left\{P_{i}: i \in I\right\}$ be a collection of disjoint $V(H)$-paths such that no $P_{i}$ has endpoints contained in a single segment of $H$. Let $G=H \cup\left(\bigcup_{i} P_{i}\right)$ and let $\gamma$ be a $\Gamma$-labeling of $G$ by a group $\Gamma$ which does not contain any elements of order two. Assume $\gamma\left(P_{i}\right)=0$ for all $i \in I$. For all $i \in I$, let $z_{i}$ be an endpoint of $P_{i}$ such that

1. $z_{i}$ is a splitting vertex of some segment of $H$, and

2. $z_{i}$ and $z_{j}$ are not contained in the same segment for all distinct $i, j \in I$.

If $|I| \geq 10^{20} k^{10}$, then $G$ contains $k$ vertex disjoint non-zero cycles.

Proof. Let $G, H, I,\left\{P_{i}: i \in I\right\},\left\{z_{i}: i \in I\right\}, \Gamma$ and $\gamma$ be as in the statement. Let

$$
|I| \geq 10^{20} k^{10} \geq 16(20)^{2} \cdot 4\left[\left(\left(16 \cdot 5^{2} 27 k^{2}\right)\left(16 \cdot 15^{2}\right)\left(10^{7} k^{8}\right)\right)+24 k^{2}\right]+16(265)^{2} k
$$

For all $i \in I$, let $w_{i}$ be the endpoint of $P_{i}$ not equal to $z_{i}$. Let $\left\{v_{i, j}: 1 \leq i \leq l, 1 \leq j \leq m\right\}$ be vertices of $H$ corresponding to the vertices of the wall. Specifically, if $X$ is the branch vertices of $H$, then $X$ has the canonical labeling. We let $Z=\bigcup_{i \in I} z_{i}$. Assume, to reach a contradiction, that $G$ does not contain $k$ disjoint non-zero cycles.

First, we observe that many of the paths $P_{i}$ have endpoints that are "distant" in the subgraph $H$. We define the function $\lambda(x, y)$ for any two vertices $x, y \in V(H)$ to be the the minimum number of branch vertices in $X$ contained in a subpath of $H$ linking $x$ and $y$. If $Y \subseteq V(H)$ is a subset of vertices, then $\lambda(x, Y)$ is $\min _{y \in Y} \lambda(x, y)$. Note that the function $\lambda$ satisfies the triangle inequality. For all integers $t \geq 5$ and vertices $v \in V(H)$, the set of vertices $x$ with $\lambda(x, v) \leq t$ is contained in a subgraph of $H$ isomorphic to a subdivision of the $(2 t+3) \times(4 t+1)$ wall. It follows that there exist at most $(2 t+3)(4 t)+(2 t+2)(2 t+1) \leq 12 t^{2}+18 t+3 \leq 16 t^{2}$ distinct segments containing a vertex $x$ with $\lambda(x, v) \leq t$.

We claim that there exists a set $I_{1} \subseteq I$ such that

1. for all $i \in I_{1}, \lambda\left(z_{i}, w_{i}\right)>32$, and,

2. for all $i, j \in I_{1}, i \neq j, \lambda\left(z_{i}, z_{j}\right)>20$.

Furthermore, the set $I_{1}$ may be chosen so that $\left|I_{1}\right| \geq\left(|I|-16(265)^{2} k\right) /\left(16 \cdot 20^{2}\right)$. To see this, let $I^{\prime} \subseteq I$ be a set of indices such that $\lambda\left(z_{i}, w_{i}\right) \leq 32$ for all $i \in I^{\prime}$. Assume, to reach a contradiction, that $\left|I^{\prime}\right| \geq 16(265)^{2} k$.

Let $i \in I^{\prime}$ and let $S$ be the segment of $H$ such that $z_{i} \in V(S)$. Let the endpoints of $S$ be $v_{a, b}$ and $v_{a^{\prime}, b^{\prime}}$. Let $X_{i}$ be defined to be $\left\{v_{i, j}:|a-i| \leq 66,|b-j| \leq 33\right\}$. This set contains every branch vertex $v_{i, j}$ with $\lambda\left(v_{i, j}, v_{a, b}\right) \leq 33$. Note that we must consider indices at distance 66 to cover the case when 
$a=1$ and the branch vertices are of the form $v_{1,2 j+1}$ for $j \geq 0$. Let $W_{i}$ be the subgraph consisting of every segment of $W$ with both endpoints in $X_{i}$. Then $W_{i}$ is a subdivision of a wall of height at most 67 and width at most 122. Moreover, by construction, both $w_{i}$ and $z_{i}$ are contained in $W_{i}$. The subgraph $W_{i} \cup P_{i}$ must then contain a non-zero theta, and consequently, a non-zero cycle.

We now show that the subgraphs $W_{i}$ are pair-wise disjoint for at least $k$ of the indices of $I^{\prime}$. Observe that for every vertex of $x \in V\left(W_{i}\right), \lambda\left(x, z_{i}\right) \leq 66+66=132$. It follows that for all $i, j \in I^{\prime}$, if $z_{i}$ and $z_{j}$ satisfy $\lambda\left(z_{i}, z_{j}\right) \geq 265$, then $W_{i}$ and $W_{j}$ are disjoint. There are less than $16(265)^{2}$ distinct segments $S$ such that $x \in V(S)$ satisfies $\lambda\left(x, z_{i}\right) \leq 265$. It follows that for at least $\left|I^{\prime}\right| /\left(16(265)^{2}\right) \geq k$ distinct indices in $I^{\prime}$, the subgraphs $W_{i}$ are pairwise disjoint. This implies that $G$ contains $k$ disjoint non-zero cycles, a contradiction.

We conclude that no such set $I^{\prime}$ of indices exists. It follows that for at least $|I|-16(265)^{2} k$ indices $i$ in $I, \lambda\left(z_{i}, w_{i}\right)>32$. Moreover, for every index $i \in I$, there exist at most $16(20)^{2}$ distinct indices $j$ such that $\lambda\left(z_{j}, z_{i}\right) \leq 20$. It follows that the desired set $I_{1}$ exists with $\left|I_{1}\right| \geq\left(|I|-16(265)^{2} k\right) /\left(16 \cdot 20^{2}\right)$.

We now define a subgraph $H^{\prime}$ of $H$ isomorphic to a subdivision of a smaller wall such that $H^{\prime}$ does not contain many of the breaking vertices $z_{i}$. In a slight abuse of notation, if $x$ and $y$ are two vertices of $H$, we use $x H y$ to refer to the unique path $P$ of $H$ linking $x$ and $y$ where every internal vertex of $P$ has degree two in $H$, whenever such a unique path exists. Let $S_{i}, 1 \leq i \leq l$, be the path $\bigcup_{j=1}^{m-1} v_{i, j} H v_{i, j+1}$. We define $R_{i}, 1 \leq i \leq(m-3) / 2$, to be the path $\bigcup_{j=1}^{l / 2} v_{2 j-1,2 i-1} H v_{2 j, 2 i-1} \cup$ $\bigcup_{j=1}^{l / 2-1} v_{2 j, 2 i} H v_{2 j+1,2 i} \cup \bigcup_{j=2^{l}-1} v_{j, 2 i-1} H v_{j, 2 i}$.

Intuitively speaking, we will select half of the paths $S_{i}$ to form the horizontal paths of our new wall, and half of the paths $R_{i}$ to form the vertical paths of our new wall. Moreover, we will do so to ensure that at least a quarter of all the vertices of $Z$ are not contained in the new wall. Towards that end, for each pair of horizontal paths $S_{i}$, we keep the one with fewer vertices $z_{j}$. Explicitly, we define the set $J_{1}$ of indices as follows. For every $i, 1 \leq i \leq l / 2$, if $\left|V\left(S_{2 i-1}\right) \cap Z\right| \leq\left|V\left(S_{2 i}\right) \cap Z\right|$, then $2 i-1 \in J_{1}$. Otherwise $2 i \in J_{1}$. If we let $Z^{\prime}=\left\{z \in Z: z \notin V\left(S_{j}\right) \quad \forall j \in J_{1}\right\}$, we see that $\left|Z^{\prime}\right| \geq|Z| / 2$. Similarly, we define $J_{2}$ as follows. For every $i, 1 \leq(m-3) / 4$, if $\left|V\left(R_{2 i-1}\right) \cap Z^{\prime}\right| \leq\left|V\left(R_{2 i}\right) \cap Z^{\prime}\right|$, then $2 i-1 \in J_{2}$. Otherwise, $2 i \in J_{2}$. The set $\bigcup_{i \in J_{1}} S_{i} \cup \bigcup_{i \in J_{2}} R_{i}$ contains a subgraph $H^{\prime}$ isomorphic to a $l / 2 \times(m-3) / 4$ wall. Observe that every segment of $H^{\prime}$ consists of at most 17 distinct segments of $H$. The remainder of the proof will show that we have many non-zero paths attaching to distinct segments of the subgraph $H^{\prime}$.

We begin with several easy observations concerning the subgraph $H^{\prime}$. By the construction of $H^{\prime}$, there exists a set $I_{2} \subseteq I_{1}$ with $\left|I_{2}\right| \geq\left|I_{1}\right| / 4$ such that $z_{i} \notin V\left(H^{\prime}\right)$ for all $i \in I_{2}$. Also, observe that for every index $i \in J_{1}$, the path $v_{i, 4} S_{i} v_{i, m-3}$ is contained in the subgraph $H^{\prime}$. It follows that for every index $i \in I_{2}, \lambda\left(z_{i}, V\left(H^{\prime}\right)\right) \leq 5$.

For every $i \in I_{2}$, we define two paths $T_{i}^{1}$ and $T_{i}^{2}$ as follows. Let $T_{i}^{1}$ be a path in $H$ linking $z_{i}$ and $V\left(H^{\prime}\right)$ intersecting as few branch vertices of $H$ as possible. If the segment of $H$ containing $Z_{i}$ is $Q$, let $C$ be a cycle of $H$ containing $Q$ and at most six branch vertices of $H$. The cycle $C$ corresponds to a facial cycle in the natural embedding of the $l \times m$ wall in the plane. Then let $T_{i}^{2}$ be a path linking $z_{i}$ and $V\left(H^{\prime}\right)$ in $T_{i}^{1} \triangle C$ the symmetric difference of $T_{i}^{1}$ and $C$. Note that $T_{i}^{1}$ contains at most five branch vertices of $H$, and $T_{i}^{2}$ contains at most 10 such branch vertices. Also, if $Q$ has endpoints equal to $u$ and $v$, then $T_{i}^{1}$ contains exactly one of the two subpaths $z_{i} Q u$ and $z_{i} Q v$, say $z_{i} Q u$, and $T_{i}^{2}$ then contains the subpath $z_{i} Q v$. By our choice of $z_{i}$ to be a splitting vertex, then either $\gamma\left(T_{i}^{1}\right) \neq \gamma\left(T_{i}^{2}\right)$ and one of the paths $T_{i}^{1}$ or $T_{i}^{2}$ is a non-zero path, or one of the $T_{i}^{1}$ and $T_{i}^{2}$ contains a non-zero segment of $H$. Also, for all $i, j \in I_{2}, i \neq j$, it follows that $T_{i}^{1} \cap T_{j}^{1}=\emptyset, T_{i}^{1} \cap T_{j}^{2}=\emptyset$ by the fact that $\lambda\left(z_{i}, z_{j}\right)>20$. 
Lemma 4.6 implies that $H$ has at most $24 k^{2}$ distinct segments of non-zero weight. It follows that there exists a subset $I_{3} \subseteq I_{2}$ with $\left|I_{3}\right| \geq\left|I_{2}\right|-24 k^{2}$ such that for all $i \in I_{3}, \gamma\left(T_{i}^{1}\right) \neq \gamma\left(T_{i}^{2}\right)$.

Assume that there exists a segment $Q$ of $H$ that is internally disjoint from $V\left(H^{\prime}\right)$ and a subset $I^{\prime \prime} \subseteq I_{3}$ with $\left|I^{\prime \prime}\right| \geq 27 k^{2}$ such that $w_{i} \in \operatorname{int}(Q)$ for all $i \in I^{\prime \prime}$. Then either $T_{i}^{1}$ or $T_{i}^{2}$ is a non-zero path with exactly one endpoint in $\operatorname{int}(Q)$ and one endpoint in $H^{\prime}$ and otherwise is disjoint from the two. Since there exists three paths, not necessarily disjoint, in $H^{\prime}$ covering every vertex of $H^{\prime}$, we see that there exists a path $Q^{\prime}$ contained in $H^{\prime}$ and $9 k^{2}$ distinct indices $i$ such that $T_{i}^{1}$ is a non-zero path with one endpoint in $Q$ and one endpoint in $Q^{\prime}$. Lemma 4.2 implies that $G$ contains $k$ disjoint non-zero cycles, a contradiction.

Recall that for any $i \in I_{3}$ there are at most $16 \cdot t^{2}$ segments containing vertices $x$ with $\lambda\left(x, w_{i}\right)<t$. It follows that for any $i \in I_{3}$, there are at most $16 \cdot 15^{2}$ distinct indices $j$ with $\lambda\left(w_{i}, z_{j}\right) \leq 15$. Also, by the above argument, there are at most $\left(16 \cdot 5^{2}\right) 27 k^{2}$ distinct indices $j$ where $w_{j}$ is contained in a segment $Q^{\prime}$ of $H$ not contained in $H^{\prime}$ with the property $\lambda\left(w_{i}, w_{j}\right) \leq 5$. we conclude that there exists a subset $I_{4} \subseteq I_{3}$ with $\left|I_{4}\right| \geq\left|I_{3}\right| /\left(\left(16 \cdot 5^{2} 27 k^{2}\right)\left(16 \cdot 15^{2}\right)\right)$ such that if $i \in I_{4}$, then for all $j \in I_{4}$,

1. $\lambda\left(w_{i}, z_{j}\right)>15$, and

2. if $w_{j}$ is contained in a segment $Q^{\prime}$ not contained in $W^{\prime}$, then $\lambda\left(w_{i}, w_{j}\right)>5$.

For every $i \in I_{4}$, let $T_{i}^{\prime}$ be a path linking $w_{i}$ and $V\left(H^{\prime}\right)$ in $H$ containing a minimal number of branch vertices of $H$. Then by our construction of $I_{4}$, the either $T_{i}^{\prime} \cup P_{i} \cup T_{i}^{1}$ or $T_{i}^{\prime} \cup P_{i} \cup T_{i}^{2}$ is non-zero path with both endpoints in $H^{\prime}$. Moreover, it is a non-zero path with endpoints in distinct segments of $H^{\prime}$. This follows from the fact that every segment of $H^{\prime}$ contains at most 17 segments of $H$, the paths $T_{i}^{1}$ and $T_{i}^{2}$ each contain at most 10 branch vertices of $H$, the path $T_{i}^{\prime}$ contains at most five branch vertices of $H$, and yet $\lambda\left(w_{i}, z_{i}\right)>32$. Given that $\left|I_{4}\right| \geq 10^{7} k^{8}$, we conclude from Lemma 4.7 that $G$ contains $k$ disjoint non-zero cycles. This final contradiction completes the proof of the lemma.

We now give the proof of Theorem 3.1.

\section{Proof. [Theorem 3.1]}

We prove Theorem 3.1 with the constant $c=10^{86}$. Let $k \geq 1$ be given and let

$$
n=10^{86} k^{87} \geq\left(2\left(10^{20} k^{10}\right)+24 k^{2}\right) 10^{65} k^{77}+10^{7} k^{8} .
$$

We assume the theorem is false. It suffices to consider a minimal $k$-counter-example $\left(H,\left\{P_{i}: 1 \leq\right.\right.$ $i \leq n\}$ ) with testifying $\Gamma$-labeling $\gamma$, and we will show that such a graph indeed does contain $k$ disjoint non-zero cycles. This contradiction would then imply the theorem. Let $l$ and $m$ be as in the definition of $k$-counter-example. Let $G=H \cup\left(\bigcup_{1}^{n} P_{i}\right)$.

We first observe that there exists a set $I \subseteq\{1,2, \ldots, n\}$ with $|I| \geq n-10^{7} k^{8}$ such that for all $i \in I$, the path $P_{i}$ does not contain a non-zero leg. This follows from Lemma 5.2. For every $i \in I$, the path $P_{i}$ must have a non-zero foot contained in some segment of $H$. Lemma 5.3 implies that for every segment $S$ of $H$, the path $P_{i}$ has a non-zero foot in $S$ for at most $10^{65} k^{77}$ distinct indices $i \in I$. Thus there exists an integer $m, m \geq|I| /\left(10^{65} k^{77}\right)=2\left(10^{20} k^{10}\right)$ and segments $S_{i}, 1 \leq i \leq m$ such that for all indices $i$ there exists some index $j \in I$ such that $S_{i}$ contains a non-zero foot of the path $P_{j}$.

We consider the segments $S_{i}$ for $1 \leq i \leq m$. For every $i$ such that $\gamma\left(S_{i}\right)=0$, it follows that there exists a breaking path $Q_{i}$ attaching to a splitting vertex on the segment $S_{i}$. By construction, for all 
$1 \leq i \leq j \leq m$, the path $Q_{i}$ is disjoint from $Q_{j}$ unless $Q_{i}=Q_{j}$ and each endpoint of $Q_{i}$ is a breaking vertex on $S_{i}$ and $S_{j}$, respectively. We conclude that there exists $10^{20} k^{10}$ disjoint breaking paths attaching to $H$. Lemma 5.10 implies that $G$ contains $k$ disjoint non-zero cycles. This contradicts our choice of $G$ to be a minimal $k$-counter-example, completing the proof of Theorem 3.1.

\section{ACKNOWLEDGEMENTS}

The author is greatly indebted to Robin Thomas and Sergey Norine for their insights and helpful discussions at the early stages of this project.

\section{References}

[1] M. Chudnovsky, J. Geelen, B. Gerards, L. Goddyn, M. Lohman and P. Seymour, Packing non-zero A-paths in group labeled graphs, Combinatorica 26 (5) (2006), 521 - 532.

[2] I. Dejter and V. Neumann-lara, Unboundedness for generalized odd cycle traversability and a Gallai conjecture, paper presented at the Fourth Caribbean Conference on Computing, Puerto Rico, 1985.

[3] P. Erdős and G. Szekeres, A Combinatorial problem in geometry, Compositio Mathematica 2 (1935), 463 - 470.

[4] T. Gallai, Maximum-minimum Sätze and verallgemeinerte Factoren von Graphen, Acta. Math. Hung. Acad. Sci. 12 (1961), 131 - 173.

[5] M. Kriesell, Disjoint A-paths in Digraphs, J. Combin. Theory, Ser. B 95 (2005) 168 - 172.

[6] P. Erdös and L. Pósa, On the maximal number of disjoint circuits of a graph, Publ. Math. Debrecen 9 (1962), 3-12.

[7] K. Kawarabayashi and A. Nakamoto, The Erdős-Pósa property for vertex- and edge-disjoint odd cycles on an orientable fixed surface, Discrete Math. 307(6) (2007), 764 - 768.

[8] K. Kawarabayashi and B. Reed, Highly parity linked graphs, to appear: Combinatorica.

[9] K. Kawarabayashi, P. Wollan Non-zero disjoint cycles in highly connected group labeled graphs, J. Combin. Theory, Ser. B 96 (2006) 754 - 757.

[10] W. Mader, Über die Maximalzahl krezungsfreier H-Wege, Archiv der Mathematik (Basel) 31 (1978), 387 - 402.

[11] R. Rado, Covering theorems for ordered sets, Proceedings of the London Math. Soc. s2-50 num. 7 (1949), $509-535$.

[12] D. Rautenbach and B. Reed, The Erdős-Pósa property for odd cycles in highly connected graphs, Combinatorica 21 (2001), 267-278.

[13] B. Reed, Mangoes and blueberries, Combinatorica 19 (1999), 267-296.

[14] N. Robertson and P. Seymour, Graph Minors X, Obstructions to a tree-decomposition, J. Combin. Theory, Ser. B 52 (1991), 153 - 190.

[15] N. Robertson, P. Seymour, and R. Thomas, Quickly excluding a planar graph, J. Combin. Thoery, Ser. B 62 (1994), 323-348.

[16] C. Thomassen, On the presence of disjoint subgraphs of a specified type, J. Graph Theory 12 (1988), 101-110.

[17] C. Thomassen, The Erdős-Pósa property for odd cycles in graphs with large connectivity, Combinatorica 21 (2001), 321-333.

[18] P. Wollan, Packing non-zero A-paths in an undirected model of group labeled graphs, to appear: J. Combin. Thoery, Ser. B. 